\title{
Synthesis of Erythritol Tetranitrate (ETN) Derivatives: Functional Group Tuning of Explosive Sensitivity
}

Nicholas Lease ${ }^{[a]} *$, Lisa M. Kay ${ }^{[a]}$, Geoffrey W. Brown ${ }^{[a]}$, David E. Chavez ${ }^{[a]}$, David Robbins ${ }^{[b]}$, Edward F.C. Byrd ${ }^{[c]}$, Gregory H. Imler ${ }^{[d]}$, Damon A. Parrish ${ }^{[d]}$ and Virginia W. Manner ${ }^{[a] *}$

[a] High Explosives Science and Technology, Los Alamos National Laboratory Los Alamos, NM 87545

[b] Detonation Science and Technology, Los Alamos National Laboratory Los Alamos, NM 87545

[c] U.S. Army Research Laboratory, Aberdeen Proving Ground, Maryland, 21005

[d] U.S. Navy Research Laboratory, Washington, DC. 20375 


\section{Table of Contents}

I. $\quad$ NMR Spectra ............................................................ S3

II. Impact Testing Data ......................................................S9

III. Friction Sensitivity Data................................................. S13

IV. Electrostatic discharge Sensitivity Data............................ S17

V. Thermal (DSC) Traces ..................................................S21

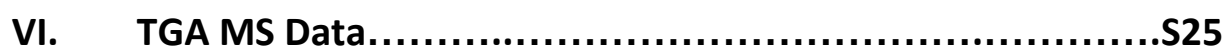

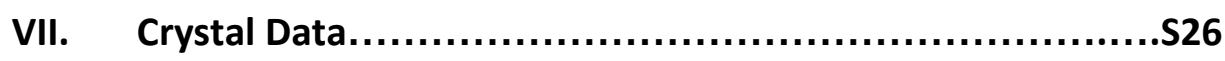




\section{NMR Spectra:}

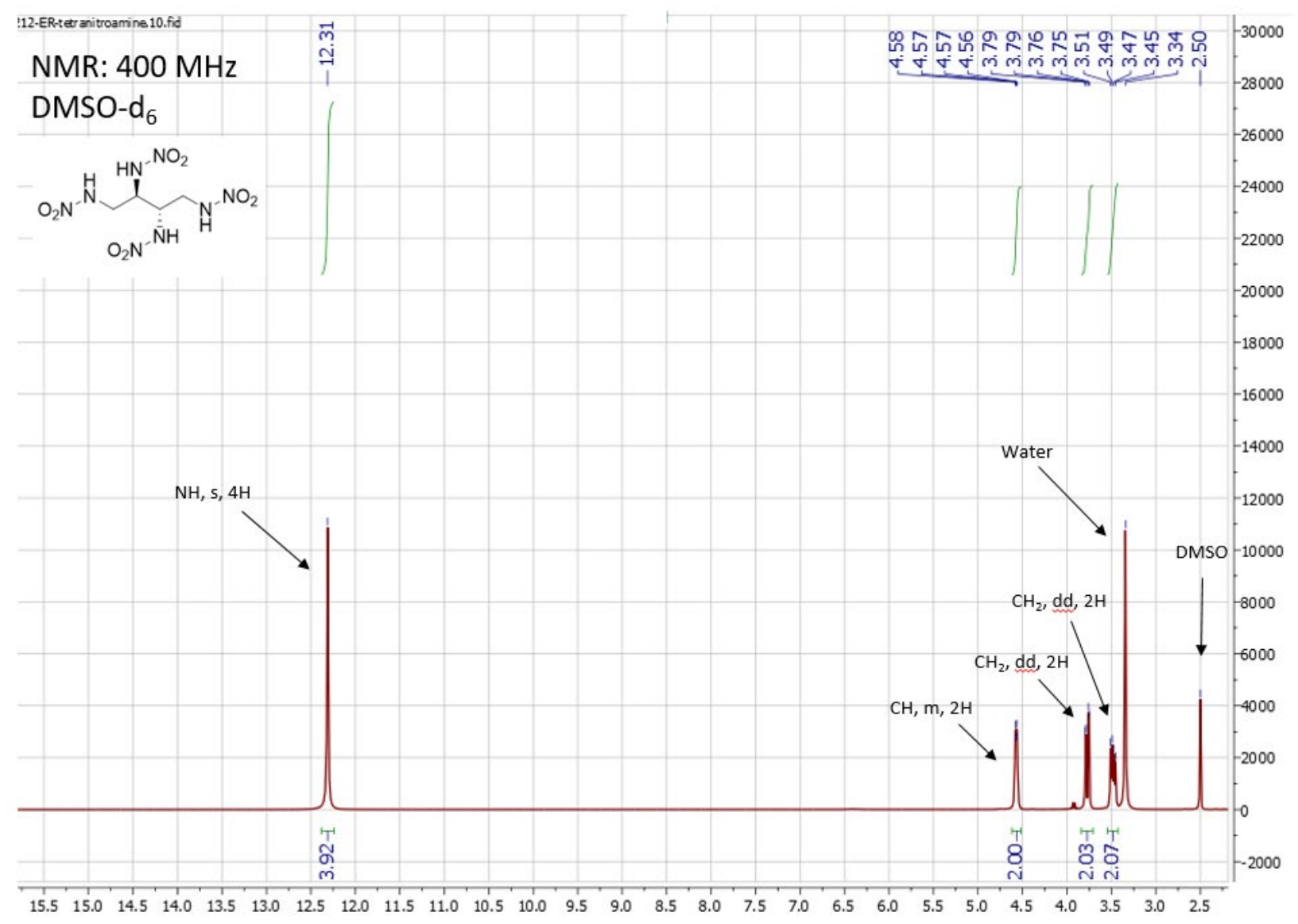

Figure S1: ${ }^{1} \mathrm{H}-\mathrm{NMR}$ of compound (4) 


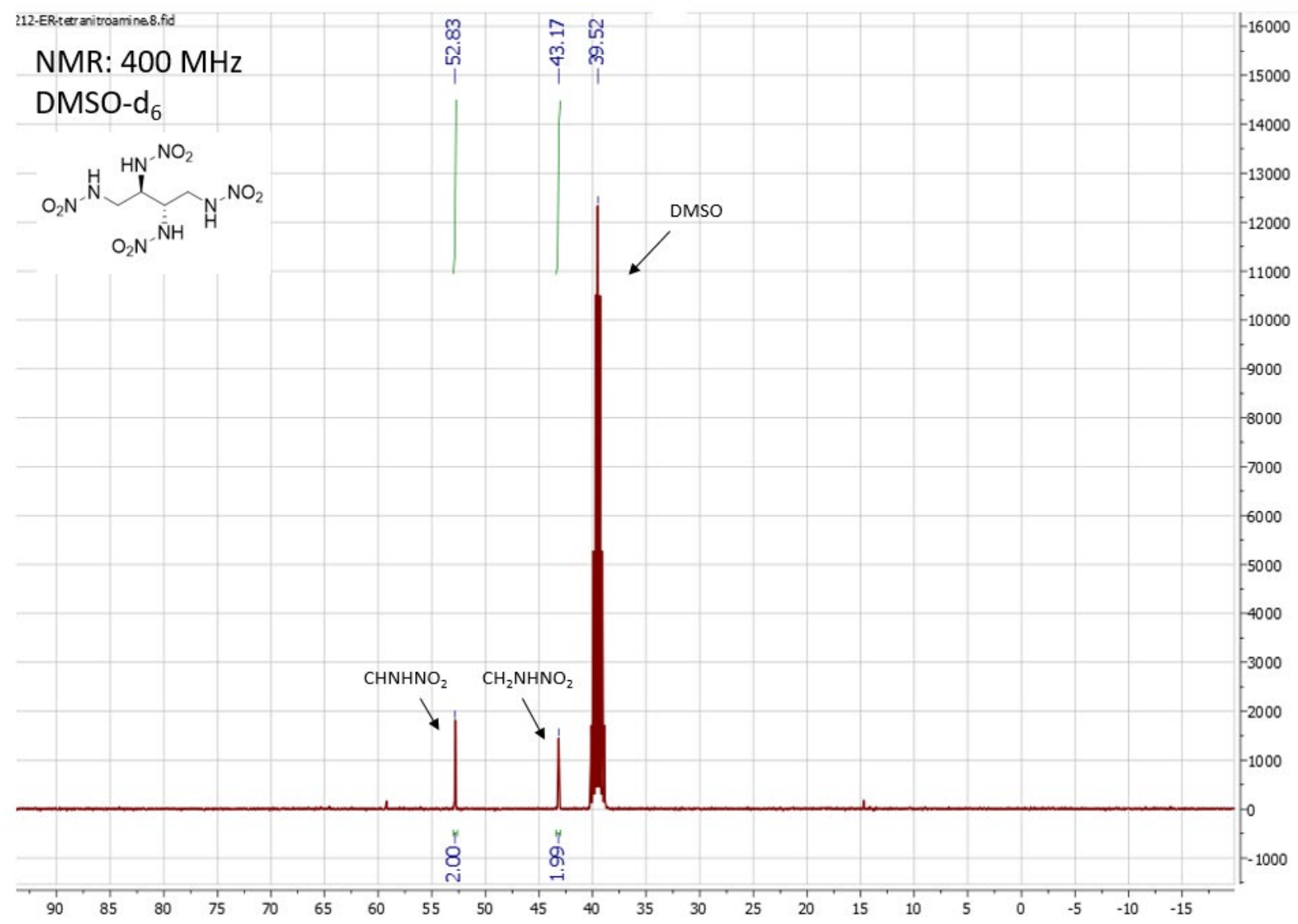

Figure S2: ${ }^{13} \mathrm{C}\left\{{ }^{1} \mathrm{H}\right\}-N M R$ of Compound (4) 


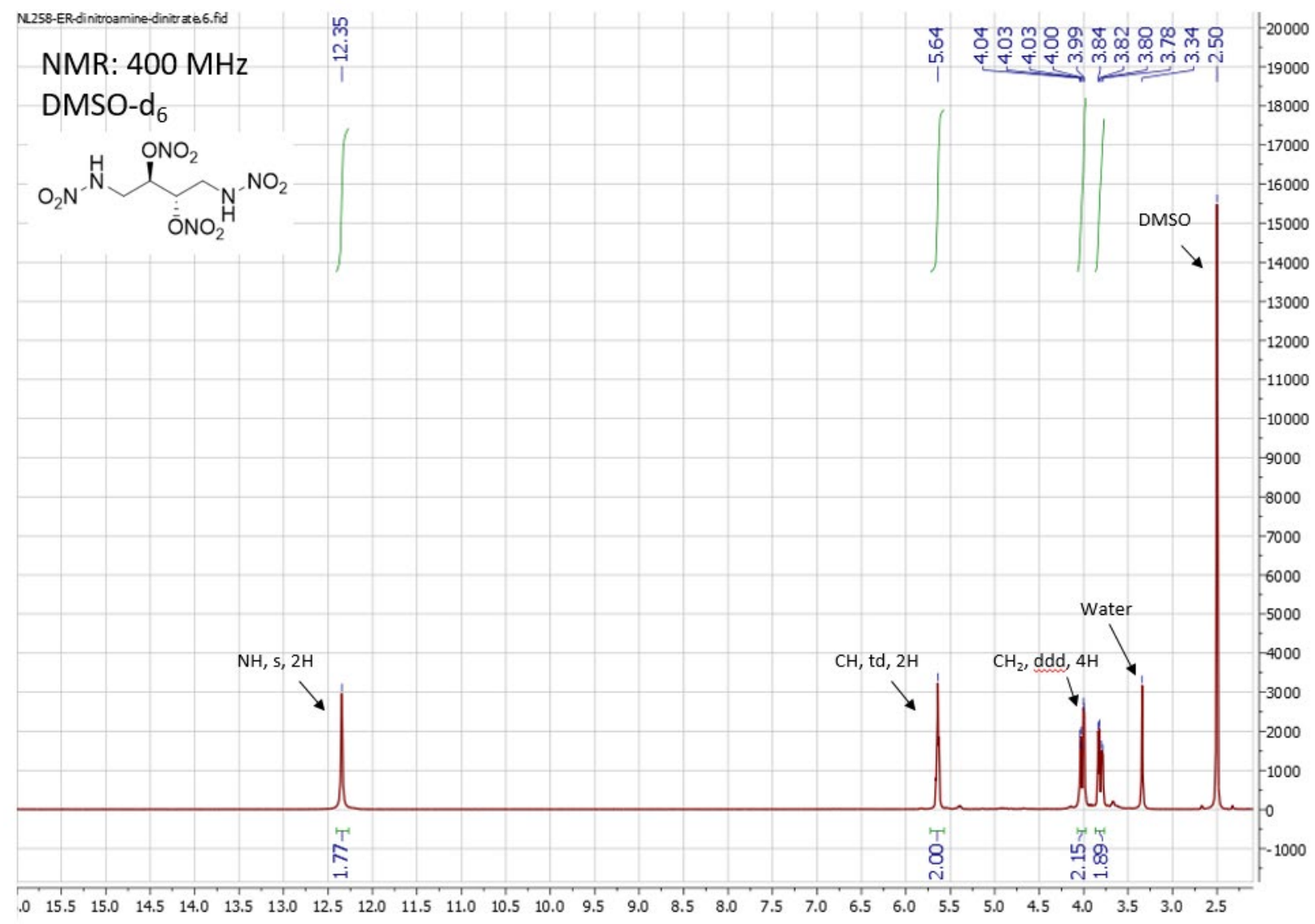

Figure S3: ${ }^{1} \mathrm{H}-\mathrm{NMR}$ of compound (8) 


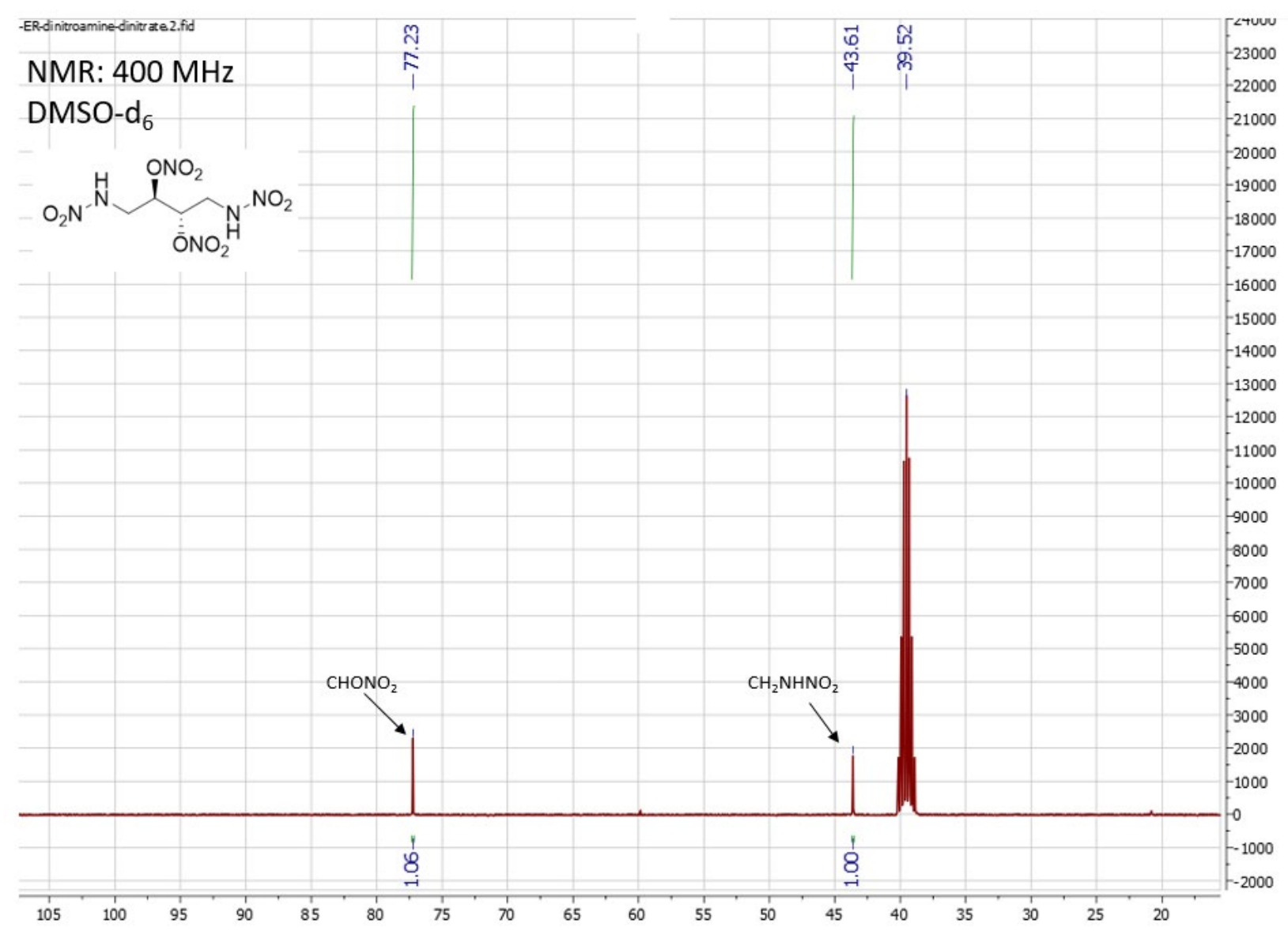

Figure S4: ${ }^{13} \mathrm{C}\left\{{ }^{1} \mathrm{H}\right\}-N M R$ of Compound (8) 


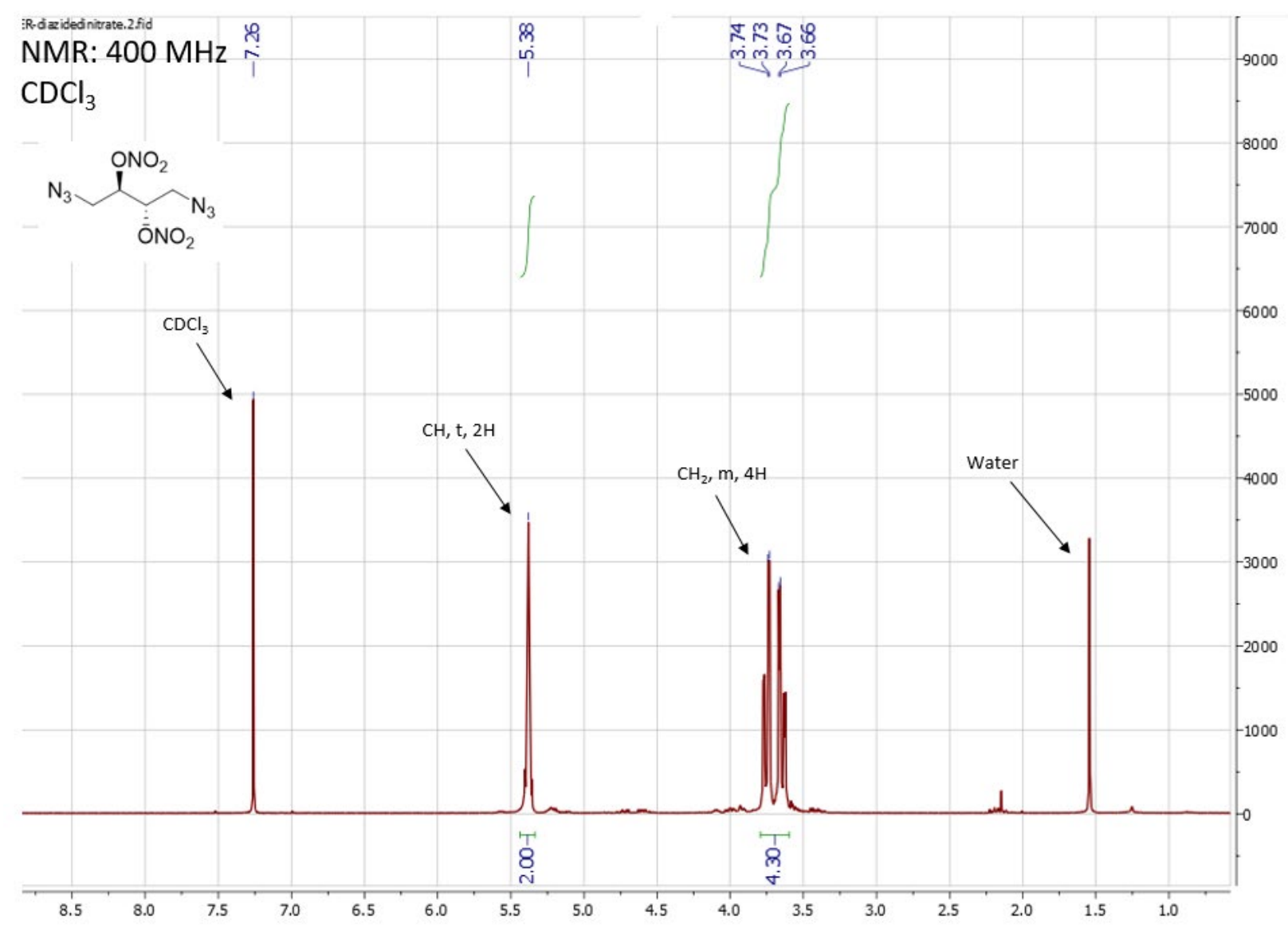

Figure S5: ${ }^{1} \mathrm{H}-\mathrm{NMR}$ of compound (9) 


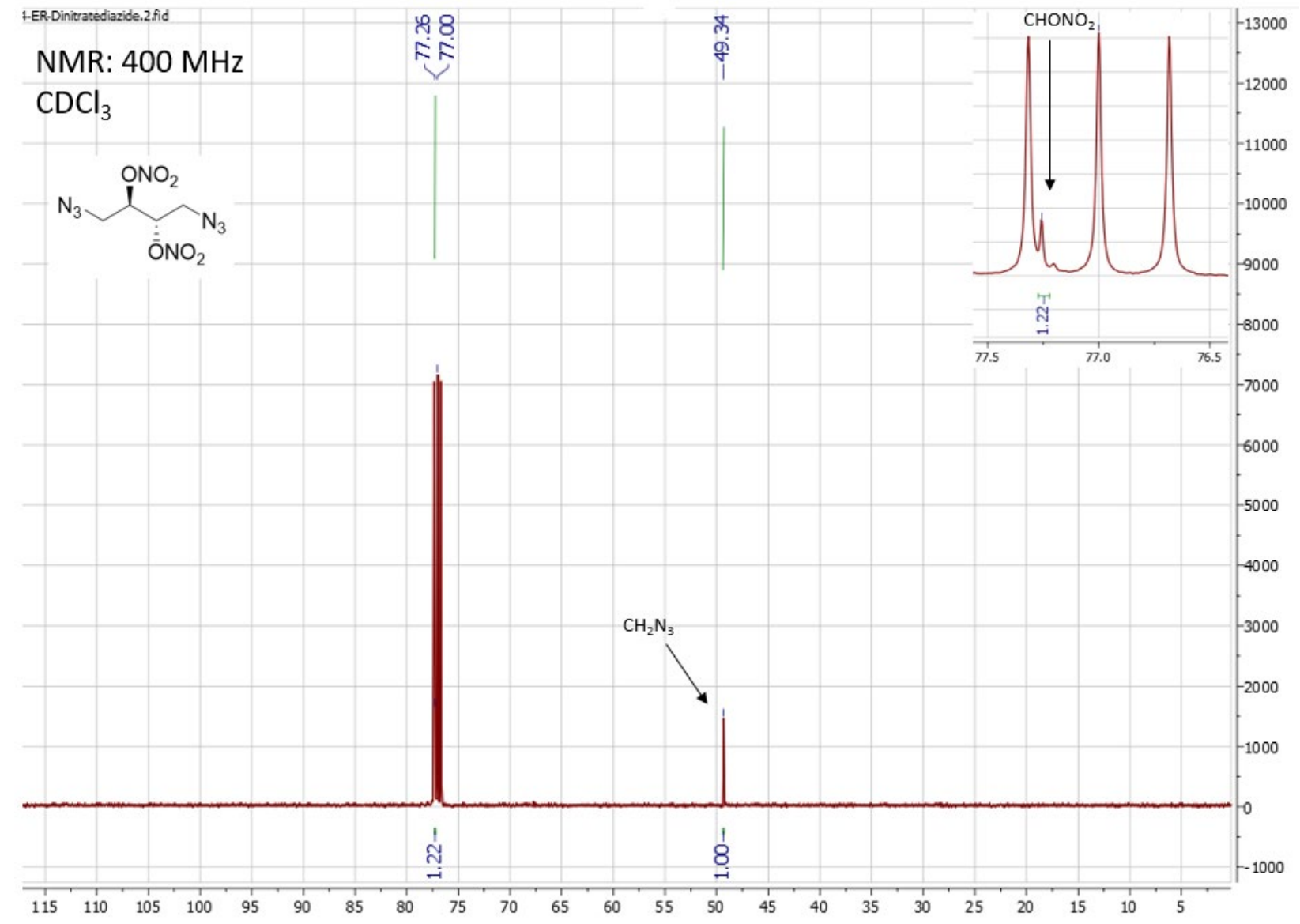

Figure S6: ${ }^{13} \mathrm{C}\left\{{ }^{1} \mathrm{H}\right\}-N M R$ of Compound (9) 


\section{Impact Testing Data:}

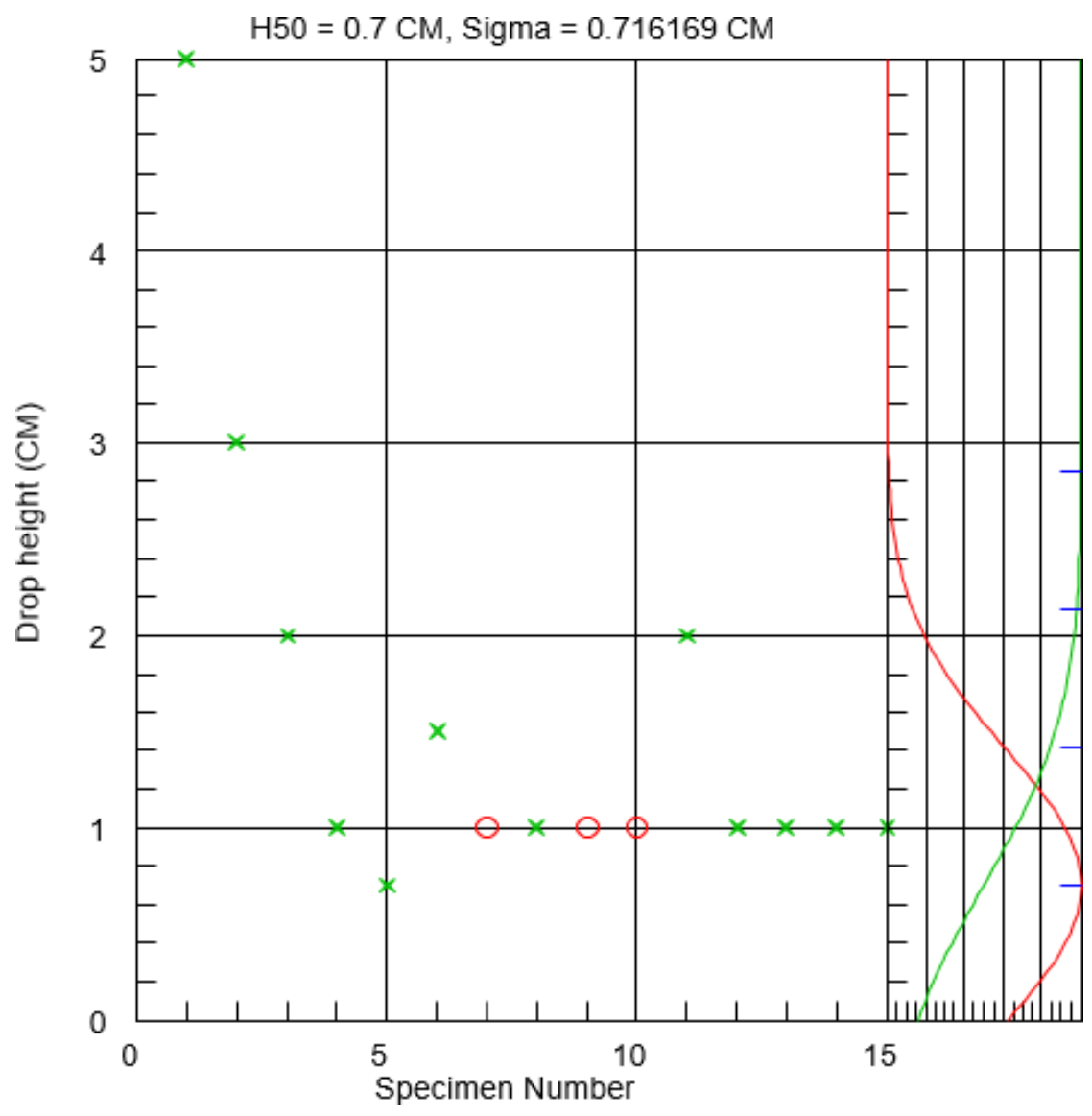

Figure S7: Impact sensitivity Neyer Plot for compound (1)

Table S1: Impact sensitivity results for compound (1)

$\begin{array}{ccccc}\text { Drop \# } & \text { Height } & \text { Go/No go } & \text { Left dB } & \text { Back dB } \\ 1 & 5 \mathrm{~cm} & 1 & 135.5 & 136.5 \\ 2 & 3 \mathrm{~cm} & 1 & 137 & 136.8 \\ 3 & 2 \mathrm{~cm} & 1 & 136.7 & 136.8 \\ 4 & 1 \mathrm{~cm} & 1 & 136.9 & 136.4 \\ 5 & 0.7 \mathrm{~cm} & 1 & 136 & 136.6 \\ 6 & 1.5 \mathrm{~cm} & 1 & 137 & 136.9 \\ 7 & 1 \mathrm{~cm} & 0 & 84.2 & 85.6 \\ 8 & 1 \mathrm{~cm} & 1 & 134.6 & 136.5 \\ 9 & 1 \mathrm{~cm} & 0 & 87.7 & 88.3 \\ 10 & 1 \mathrm{~cm} & 0 & 86.7 & 86.6 \\ 11 & 2 \mathrm{~cm} & 1 & 135.5 & 136.4 \\ 12 & 1 \mathrm{~cm} & 1 & 135.8 & 136.7 \\ 13 & 1 \mathrm{~cm} & 1 & 135.7 & 136.5 \\ 14 & 1 \mathrm{~cm} & 1 & 135.9 & 136.4 \\ 15 & 1 \mathrm{~cm} & 1 & 135.7 & 136.6\end{array}$

Comments (conditions after drop) nothing left louder than the last one, nothing left nothing left

lost magnet - flattened liquid, went anyway nothing left

lost magnet - flattened liquid, went anyway new magnet arrangement, all sample left new magnet arrangement; no sample left All sample left All sample left nothing left nothing left nothing left nothing left nothing left 


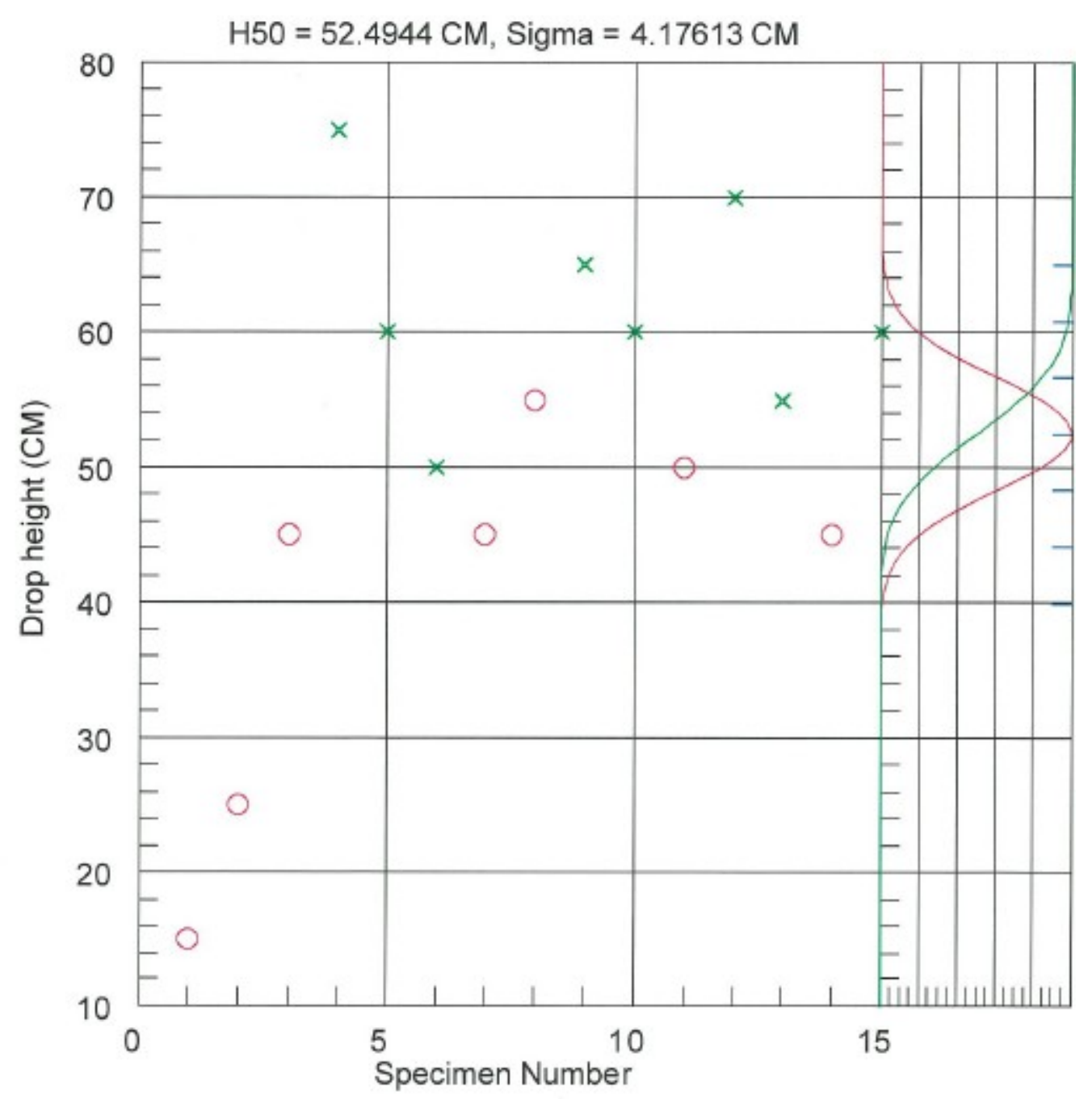

Figure S8: Impact sensitivity Neyer Plot for compound (4)

Table S2: Impact sensitivity results for compound (4)

\begin{tabular}{|c|c|c|c|c|c|}
\hline Drop \# & Height & Go/No go & Left $d B$ & Back dB & Comments (conditions after drop) \\
\hline 1 & $15 \mathrm{~cm}$ & 0 & 98.1 & 102.9 & All HE remained \\
\hline 2 & $25 \mathrm{~cm}$ & 0 & 97.5 & 101.1 & All HE remained \\
\hline 3 & $45 \mathrm{~cm}$ & 0 & 102.1 & 104.7 & All HE remained \\
\hline 4 & $75 \mathrm{~cm}$ & 1 & 118.3 & 122.5 & All HE remained \\
\hline 5 & $60 \mathrm{~cm}$ & 1 & 123.6 & 125.1 & All HE remained \\
\hline 6 & $50 \mathrm{~cm}$ & 1 & 116.8 & 118.5 & All HE remained \\
\hline 7 & $45 \mathrm{~cm}$ & 0 & 99.8 & 101.7 & All HE remained \\
\hline 8 & $55 \mathrm{~cm}$ & 0 & 110.9 & 117.5 & All HE remained \\
\hline 9 & $65 \mathrm{~cm}$ & 1 & 124.8 & 126.3 & All HE remained \\
\hline 10 & $60 \mathrm{~cm}$ & 1 & 122.1 & 123.2 & All HE remained \\
\hline 11 & $50 \mathrm{~cm}$ & 0 & 110.3 & 112.1 & All HE remained \\
\hline 12 & $70 \mathrm{~cm}$ & 1 & 127.4 & 128.2 & All HE remained \\
\hline 13 & $55 \mathrm{~cm}$ & 1 & 125.6 & 127.2 & All $\mathrm{HE}$ remained \\
\hline 14 & $45 \mathrm{~cm}$ & 0 & 98.7 & 100.1 & All HE remained \\
\hline 15 & $60 \mathrm{~cm}$ & 1 & 126.4 & 127.9 & All HE remained \\
\hline
\end{tabular}




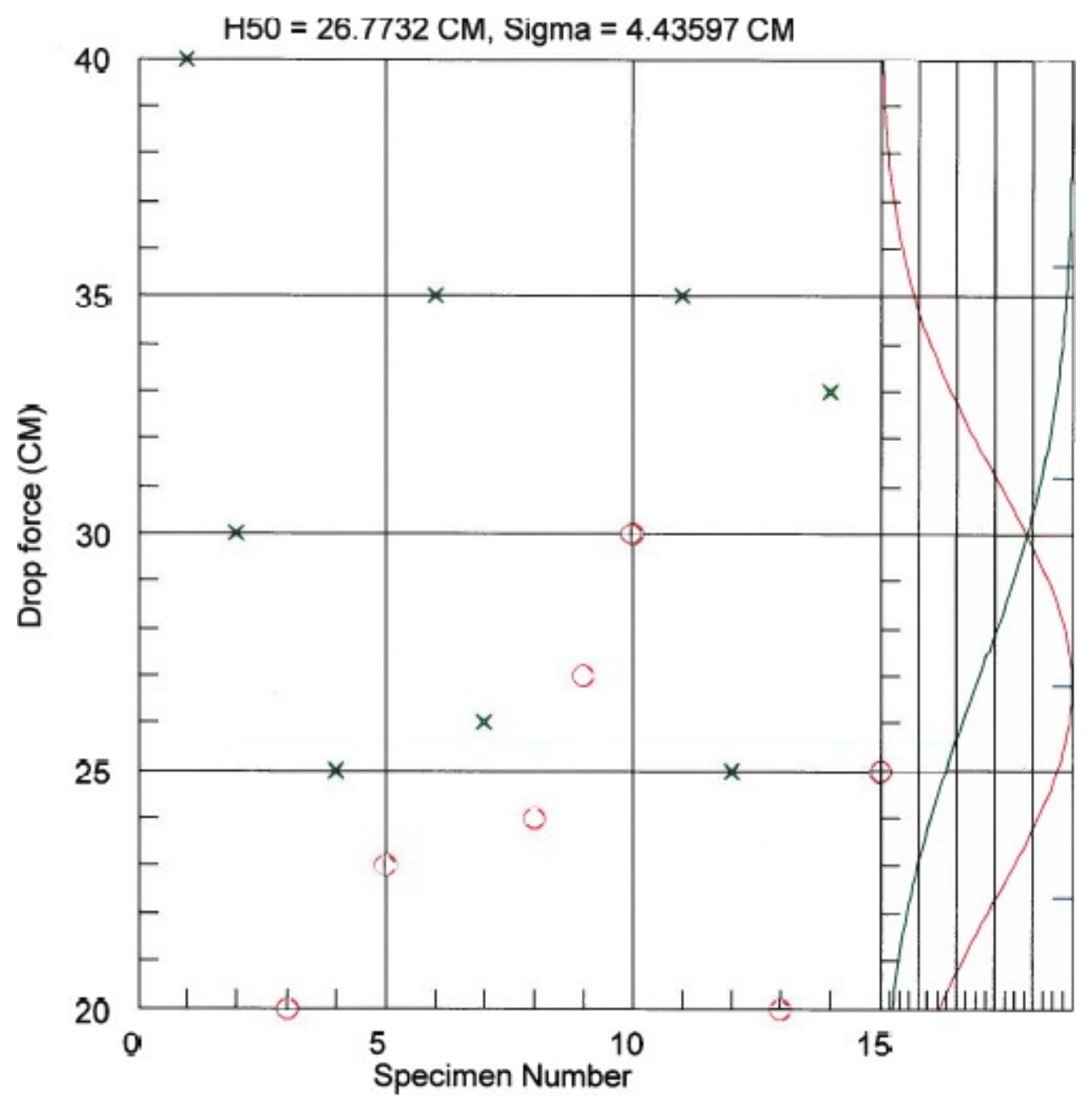

Figure S9: Impact sensitivity Neyer Plot for compound (8)

Table S3: Impact sensitivity results for compound (8)

\begin{tabular}{|c|c|c|c|c|}
\hline Drop \# & Height & Go/No go & Left dB & Back dB \\
\hline 1 & $40 \mathrm{~cm}$ & 1 & 126.2 & 127.8 \\
\hline 2 & $30 \mathrm{~cm}$ & 1 & 119.8 & 120.4 \\
\hline 3 & $20 \mathrm{~cm}$ & 0 & 96.2 & 99.2 \\
\hline 4 & $25 \mathrm{~cm}$ & 1 & 118.7 & 118.8 \\
\hline 5 & $23 \mathrm{~cm}$ & 0 & 96.1 & 99.6 \\
\hline 6 & $35 \mathrm{~cm}$ & 1 & 126.9 & 127.8 \\
\hline 7 & $26 \mathrm{~cm}$ & 1 & 124.9 & 124.5 \\
\hline 8 & $24 \mathrm{~cm}$ & 0 & 96.5 & 100.1 \\
\hline 9 & $27 \mathrm{~cm}$ & 0 & 97.8 & 100.8 \\
\hline 10 & $30 \mathrm{~cm}$ & 0 & 98.6 & 101.5 \\
\hline 11 & $35 \mathrm{~cm}$ & 1 & 122.7 & 124.9 \\
\hline 12 & $25 \mathrm{~cm}$ & 1 & 119.2 & 118.4 \\
\hline 13 & $20 \mathrm{~cm}$ & 0 & 95 & 99.5 \\
\hline 14 & $33 \mathrm{~cm}$ & 1 & 123 & 122.5 \\
\hline 15 & $25 \mathrm{~cm}$ & 0 & 96.7 & 99.9 \\
\hline
\end{tabular}

Comments (conditions after drop)

Go, HE left over solid gooey when smacked Go, HE left over solid gooey when smacked No go, HE left over, becomes gooey Go, HE left over solid gooey when smacked No go, HE left over, becomes gooey Go, HE left over solid gooey when smacked Go, HE left over solid gooey when smacked No go, HE left over, becomes gooey No go, HE left over, becomes gooey No go, HE left over, becomes gooey Go, HE left over solid gooey when smacked Go, HE left over solid gooey when smacked No go, HE left over, becomes gooey Go, HE left over solid gooey when smacked No go, HE left over, becomes gooey 


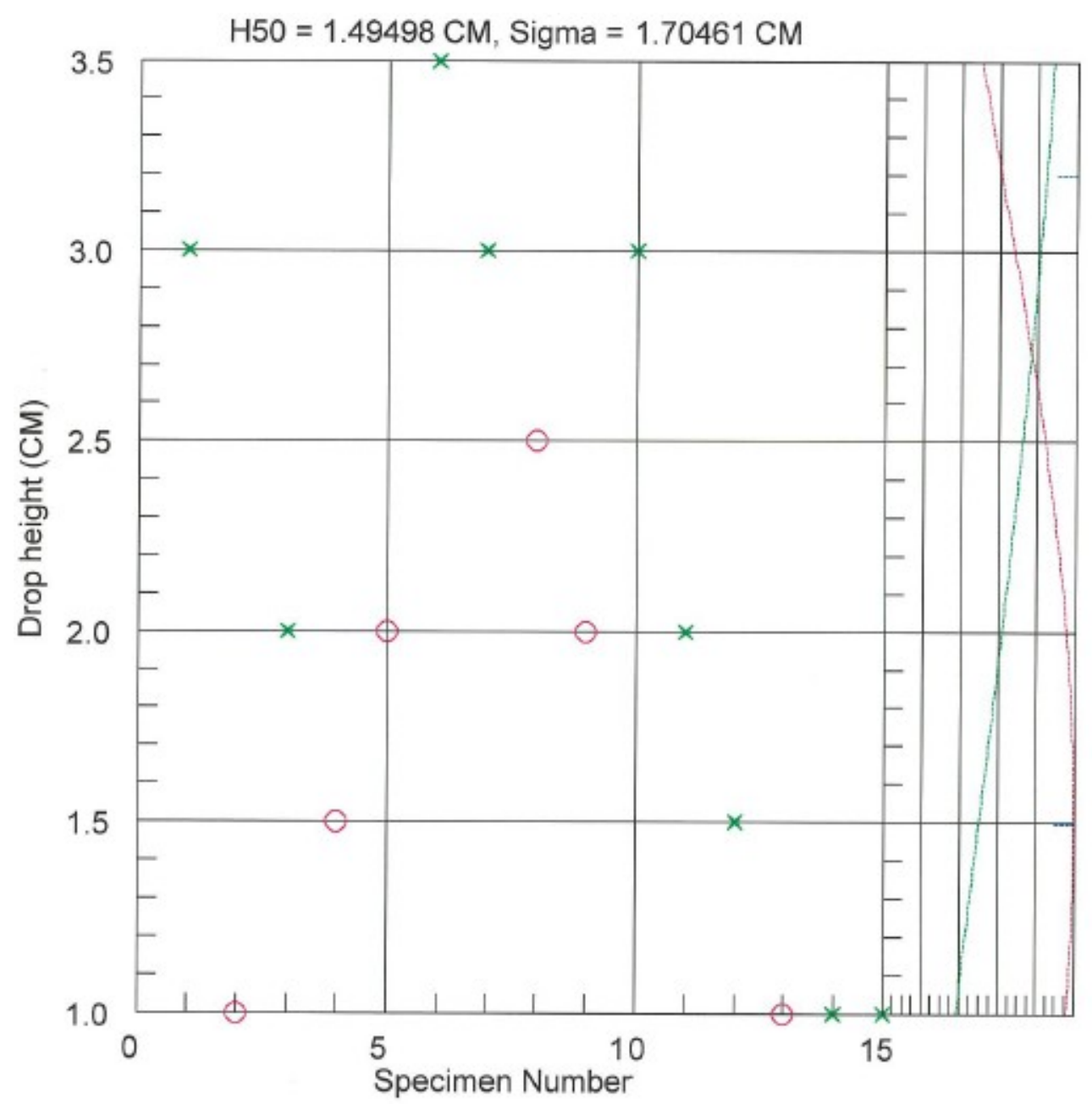

Figure S10: Impact sensitivity Neyer Plot for compound (9)

Table S4: Impact sensitivity results for compound (9)

\begin{tabular}{ccccc} 
& & & \multicolumn{2}{c}{ Sound Meters } \\
Drop \# & Height & Go/No go & Left dB & Back dB \\
1 & $3 \mathrm{~cm}$ & 1 & 136.7 & 137 \\
2 & $1 \mathrm{~cm}$ & 0 & 89.7 & 92.3 \\
3 & $2 \mathrm{~cm}$ & 1 & 136.3 & 137.1 \\
4 & $1.5 \mathrm{~cm}$ & 0 & 89.4 & 91.9 \\
5 & $2 \mathrm{~cm}$ & 0 & 88.8 & 90.8 \\
6 & $3.5 \mathrm{~cm}$ & 1 & 135.2 & 136.3 \\
7 & $3 \mathrm{~cm}$ & 1 & 136.5 & 137.1 \\
8 & $2.5 \mathrm{~cm}$ & 0 & 90.9 & 91.5 \\
9 & $2 \mathrm{~cm}$ & 0 & 91 & 91.7 \\
10 & $3 \mathrm{~cm}$ & 1 & 136.7 & 136.7 \\
11 & $2 \mathrm{~cm}$ & 1 & 137 & 136.8 \\
12 & $1.5 \mathrm{~cm}$ & 1 & 137.1 & 137 \\
13 & $1 \mathrm{~cm}$ & 0 & 90.3 & 91.6 \\
14 & $1 \mathrm{~cm}$ & 1 & 136.9 & 136.8 \\
15 & $1 \mathrm{~cm}$ & 1 & 136 & 136.9
\end{tabular}

Comments (conditions after drop) Go nothing left

No go, all HE remained

Go nothing left

No go, all HE remained

No go, all HE remained

Go nothing left

Go nothing left

No go, all $\mathrm{HE}$ remained

No go, all HE remained

Go nothing left, anvil* and magnets broke

Go nothing left

Go nothing left

No go, all HE remained

Go nothing left

Go nothing left 
Friction Sensitivity Data:

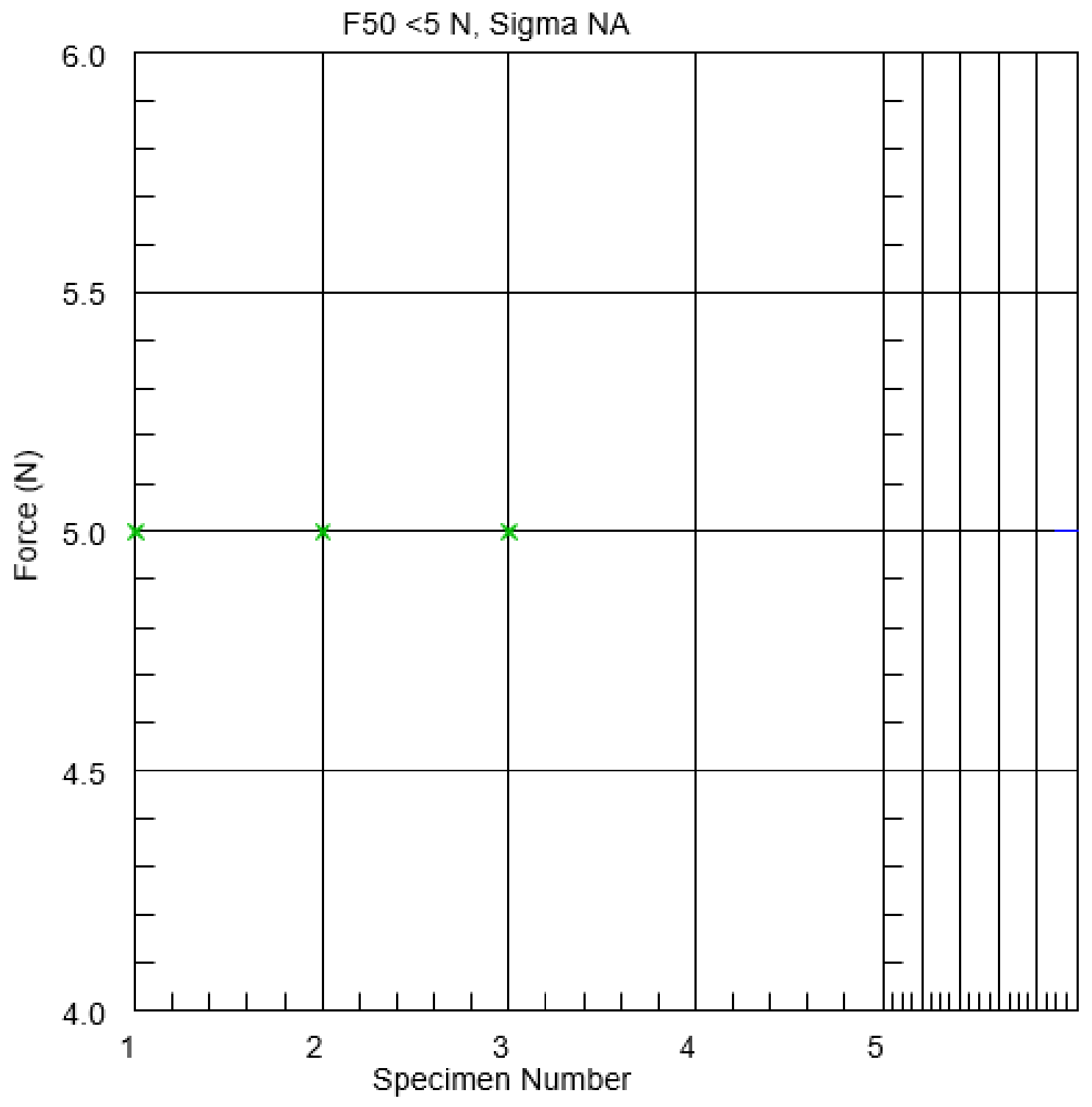

Figure S11: Friction sensitivity Neyer Plot for compound (1) 


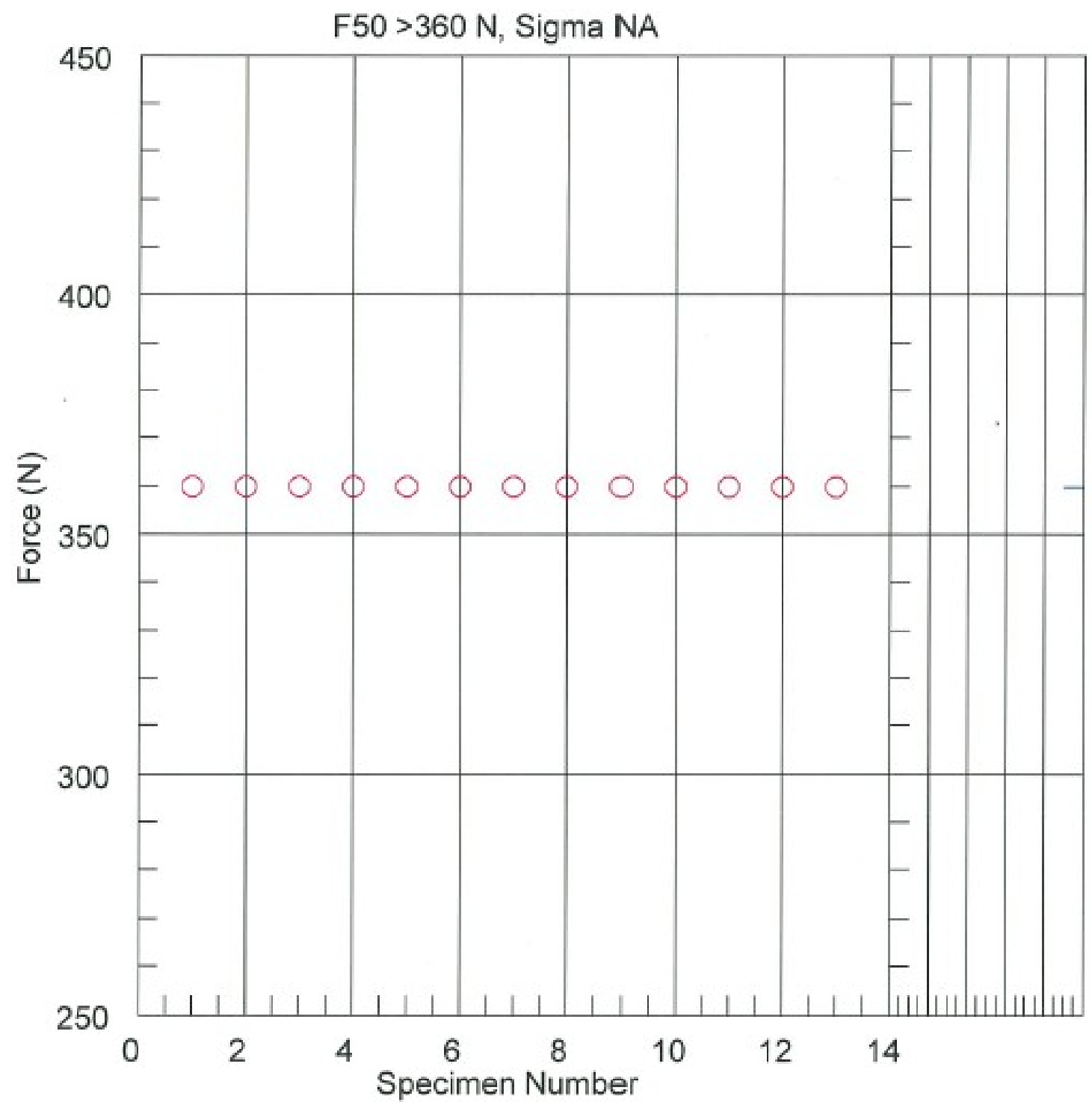

Figure S12: Friction sensitivity Neyer Plot for compound (4) 


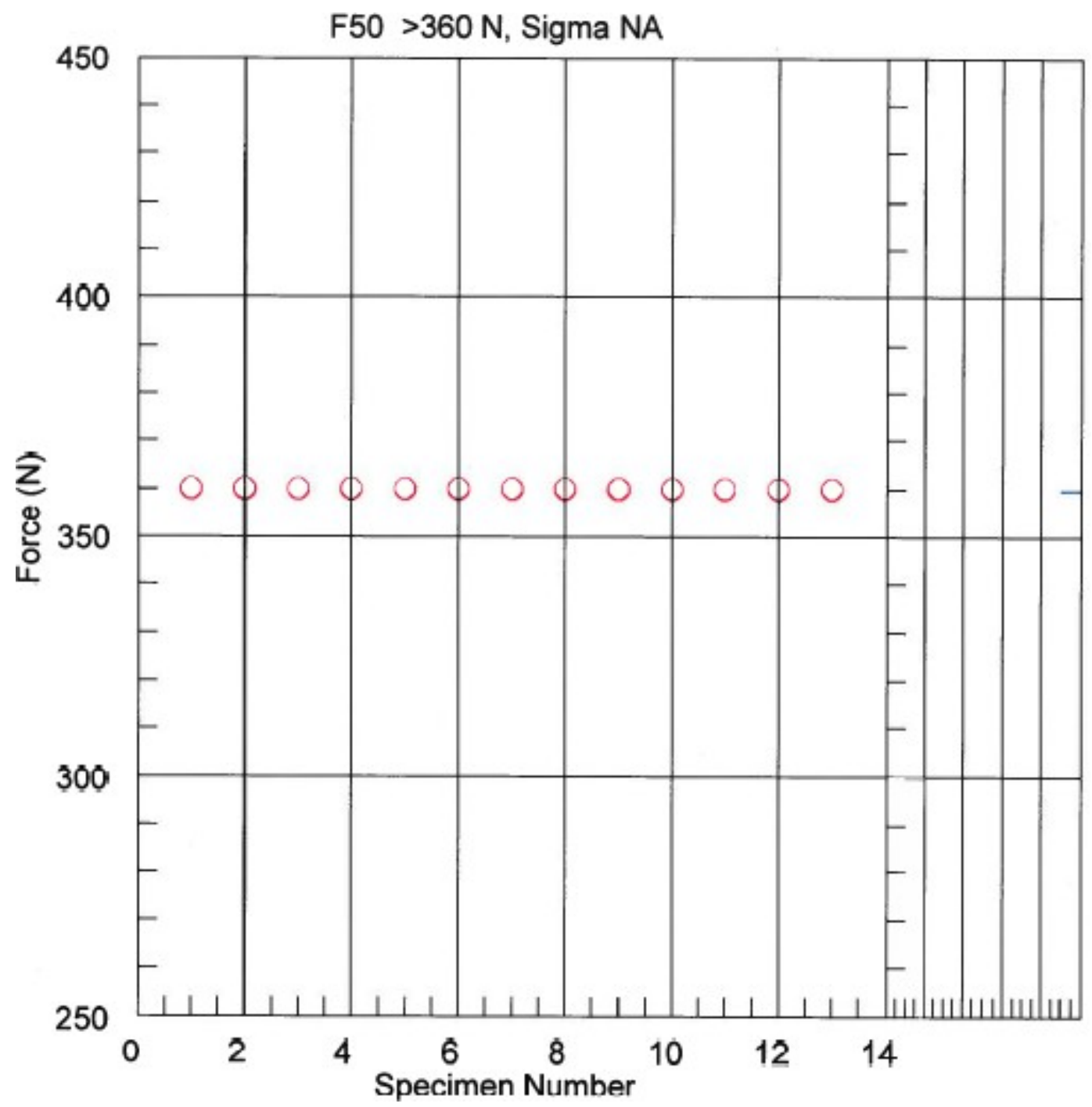

Figure S13: Friction sensitivity Neyer Plot for compound (8) 


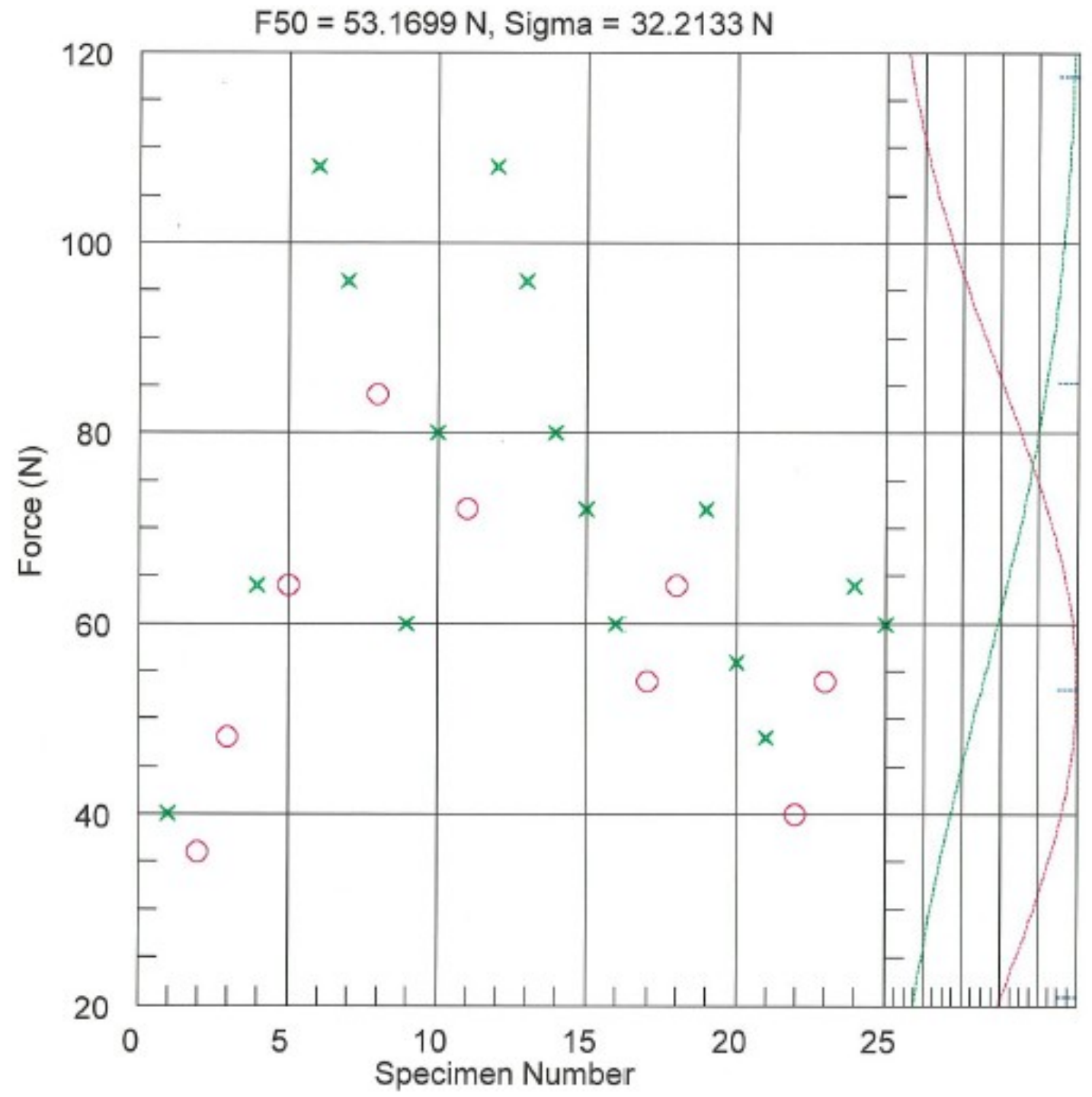

Figure S14: Friction sensitivity Neyer Plot for compound (9) 


\section{Electrostatic Discharge Sensitivity Data:}

\begin{tabular}{|r|c|c|c|c|c|c|c|c|c|c|}
\hline Discharge Level & 1 & 2 & 3 & 4 & 5 & 6 & 7 & 8 & 9 & 10 \\
\hline $\mathbf{0 . 2 5}$ Joules & $\mathrm{E}$ & & & & & & & & & \\
\hline $\mathbf{0 . 1 2 5}$ Joules & $\mathrm{N}$ & $\mathrm{N}$ & $\mathrm{N}$ & $\mathrm{N}$ & $\mathrm{N}$ & $\mathrm{N}$ & $\mathrm{N}$ & $\mathrm{N}$ & $\mathrm{N}$ & $\mathrm{N}$ \\
\hline $\mathbf{0 . 0 6 2 5}$ Joules & & & & & & & & & & \\
\hline $\mathbf{0 . 0 2 5}$ Joules & & & & & & & & & & \\
\hline
\end{tabular}

\begin{tabular}{|r|c|c|c|c|c|c|c|c|c|c|}
\hline Discharge Level & 11 & 12 & 13 & 14 & 15 & 16 & 17 & 18 & 19 & 20 \\
\hline $\mathbf{0 . 2 5}$ Joules & & & & & & & & & & \\
\hline $\mathbf{0 . 1 2 5}$ Joules & $\mathrm{N}$ & $\mathrm{N}$ & $\mathrm{N}$ & $\mathrm{N}$ & $\mathrm{N}$ & $\mathrm{N}$ & $\mathrm{N}$ & $\mathrm{N}$ & $\mathrm{N}$ & $\mathrm{N}$ \\
\hline $\mathbf{0 . 0 6 2 5}$ Joules & & & & & & & & & & \\
\hline $\mathbf{0 . 0 2 5}$ Joules & & & & & & & & & & \\
\hline
\end{tabular}

Type of Test

Screen Test: Energy level set to 0.25 J (ten-times human electrostatic discharge)-Switch A set to position 5 , Switch B set to position 1 - for 13 tests.

TIL(Threshold of Initiation Level): Standard energy level (Switch B set to position 1) at which 20 consecutive "no-go" tests are observed.

\begin{tabular}{|r|c|}
\hline TIL $(\mathrm{J}):$ & 0.125 \\
\hline SCREEN $(\mathrm{J}):$ & - \\
\hline
\end{tabular}

Table S5: Electrostatic discharge data for compound (1) 
Test Results

\begin{tabular}{|r|c|c|c|c|c|c|c|c|c|c|}
\hline Discharge Level & 1 & 2 & 3 & 4 & 5 & 6 & 7 & 8 & 9 & 10 \\
\hline \hline 0.25 Joules & $\mathrm{N}$ & $\mathrm{N}$ & $\mathrm{N}$ & $\mathrm{N}$ & $\mathrm{N}$ & $\mathrm{N}$ & $\mathrm{N}$ & $\mathrm{N}$ & $\mathrm{N}$ & $\mathrm{N}$ \\
\hline $\mathbf{0 . 1 2 5}$ Joules & & & & & & & & & & \\
\hline $\mathbf{0 . 0 6 2 5}$ Joules & & & & & & & & & & \\
\hline 0.025 Joules & & & & & & & & & & \\
\hline
\end{tabular}

Test Results (continued)

\begin{tabular}{|r|c|c|c|c|c|c|c|c|c|c|}
\hline Discharge Level & 11 & 12 & 13 & 14 & 15 & 16 & 17 & 18 & 19 & 20 \\
\hline \hline 0.25 Joules & $\mathrm{N}$ & $\mathrm{N}$ & $\mathrm{N}$ & & & & & & & \\
\hline $\mathbf{0 . 1 2 5}$ Joules & & & & & & & & & & \\
\hline $\mathbf{0 . 0 6 2 5}$ Joules & & & & & & & & & & \\
\hline $\mathbf{0 . 0 2 5}$ Joules & & & & & & & & & & \\
\hline
\end{tabular}

Iype of Test

Screen Test: Energy level set to $0.25 \mathrm{~J}$ (ten-times human electrostatic discharge) - Switch A set to position 5, Switch B set to position 1 - for 13 tests.

TIL (Threshold of Initiaiton Level): Standard energy level (Switch B set to position 1) at which 20 consecutive "no-go" tests are observed.

\begin{tabular}{|r|c|}
\hline TIL $(J):$ & - \\
\hline SCREEN $(J):$ & 0.25 \\
\hline
\end{tabular}

Table S6: Electrostatic discharge data for compound (4) 
Test Results

\begin{tabular}{|r|r|r|r|r|r|r|r|r|r|r|}
\hline Discharge Level & 1 & 2 & 3 & 4 & 5 & 6 & 7 & 8 & 9 & 10 \\
\hline \hline 0.25 Joules & $\mathrm{N}$ & $\mathrm{N}$ & $\mathrm{N}$ & $\mathrm{N}$ & $\mathrm{N}$ & $\mathrm{N}$ & $\mathrm{N}$ & $\mathrm{N}$ & $\mathrm{N}$ & $\mathrm{N}$ \\
\hline $\mathbf{0 . 1 2 5}$ Joules & & & & & & & & & & \\
\hline 0.0625 Joules & & & & & & & & & & \\
\hline 0.025 Joules & & & & & & & & & & \\
\hline
\end{tabular}

Table

s7:

Test Results (continued)

\begin{tabular}{|r|c|c|c|c|c|c|c|c|c|c|}
\hline Discharge Level & 11 & 12 & 13 & 14 & 15 & 16 & 17 & 18 & 19 & 20 \\
\hline \hline 0.25 Joules & $\mathrm{N}$ & $\mathrm{N}$ & $\mathrm{N}$ & & & & & & & \\
\hline $\mathbf{0 . 1 2 5}$ Joules & & & & & & & & & & \\
\hline $\mathbf{0 . 0 6 2 5}$ Joules Joules & & & & & & & & & & \\
\hline
\end{tabular}

Type of Test

Screen Test Energy level set to 0.25 J (ten-times humsn electrostatic discharge) - Switch A set to position 5, Switch B set to position 1 - for 13 tests.

TIL (Threshold of Initiaiton Level): Standard energy level (Switch B set to position 1) at which 20 consecutive "no-go" tests are observed.

\begin{tabular}{|r|c|}
\hline TIL $(\mathrm{J}):$ & - \\
\hline SCREEN $(\mathrm{J}):$ & 0.25 \\
\hline
\end{tabular}

Table S7: Electrostatic discharge data for compound (8) 
Test Results

\begin{tabular}{|r|c|c|c|c|c|c|c|c|c|c|}
\hline \multicolumn{1}{|c|}{ Discharge Level } & 1 & 2 & 3 & 4 & 5 & 6 & 7 & 8 & 9 & 10 \\
\hline $\mathbf{0 . 2 5}$ Joules & $\mathrm{E}$ & & & & & & & & & \\
\hline $\mathbf{0 . 1 2 5}$ Joules & $\mathrm{N}$ & $\mathrm{N}$ & $\mathrm{E}$ & & & & & & & \\
\hline $\mathbf{0 . 0 6 2 5}$ Joules & $\mathrm{N}$ & $\mathrm{N}$ & $\mathrm{N}$ & $\mathrm{N}$ & $\mathrm{N}$ & $\mathrm{N}$ & $\mathrm{N}$ & $\mathrm{N}$ & $\mathrm{N}$ & $\mathrm{N}$ \\
\hline $\mathbf{0 . 0 2 5}$ Joules & & & & & & & & & & \\
\hline
\end{tabular}

Test Results (continued)

\begin{tabular}{|r|c|c|c|c|c|c|c|c|c|c|}
\hline Discharge Level & 11 & 12 & 13 & 14 & 15 & 16 & 17 & 18 & 19 & 20 \\
\hline 0.25 Joules & & & & & & & & & & \\
\hline $\mathbf{0 . 1 2 5}$ Joules & & & & & & & & & & \\
\hline $\mathbf{0 . 0 6 2 5}$ Joules & $\mathrm{N}$ & $\mathrm{N}$ & $\mathrm{N}$ & $\mathrm{N}$ & $\mathrm{N}$ & $\mathrm{N}$ & $\mathrm{N}$ & $\mathrm{N}$ & $\mathrm{N}$ & $\mathrm{N}$ \\
\hline $\mathbf{0 . 0 2 5}$ Joules & & & & & & & & & & \\
\hline
\end{tabular}

Ixpe of Test

Screen Test: Energy level set to $0.25 \mathrm{~J}$ (ten-imes human electrostatic dischargo) - Switch A set to position 5, Swìch B pot to position 1 - for 13 tests

TIL (Threshold of Initaiton Levei): Standard energy level (Switch B set to position 1) at which 20 consecutive "no-go" tests are cbserved.

\begin{tabular}{|r|c|}
\hline TIL $(J):$ & 0.0625 \\
\hline SCREEN $(J):$ & - \\
\hline
\end{tabular}

Table S8: Electrostatic discharge data for compound (9) 


\section{Thermal (DSC) Traces:}

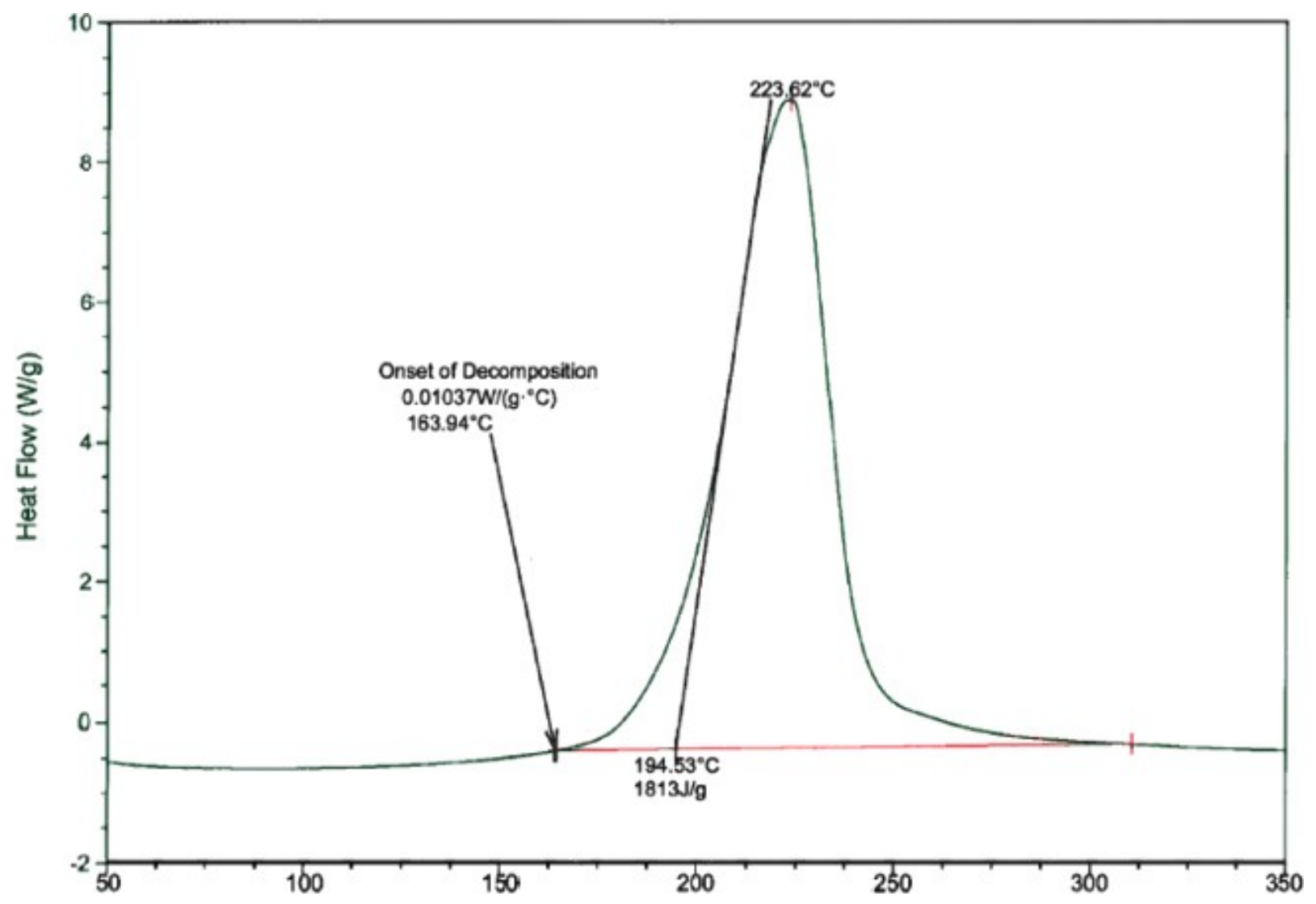

Figure S15: DSC trace of compound (1) 


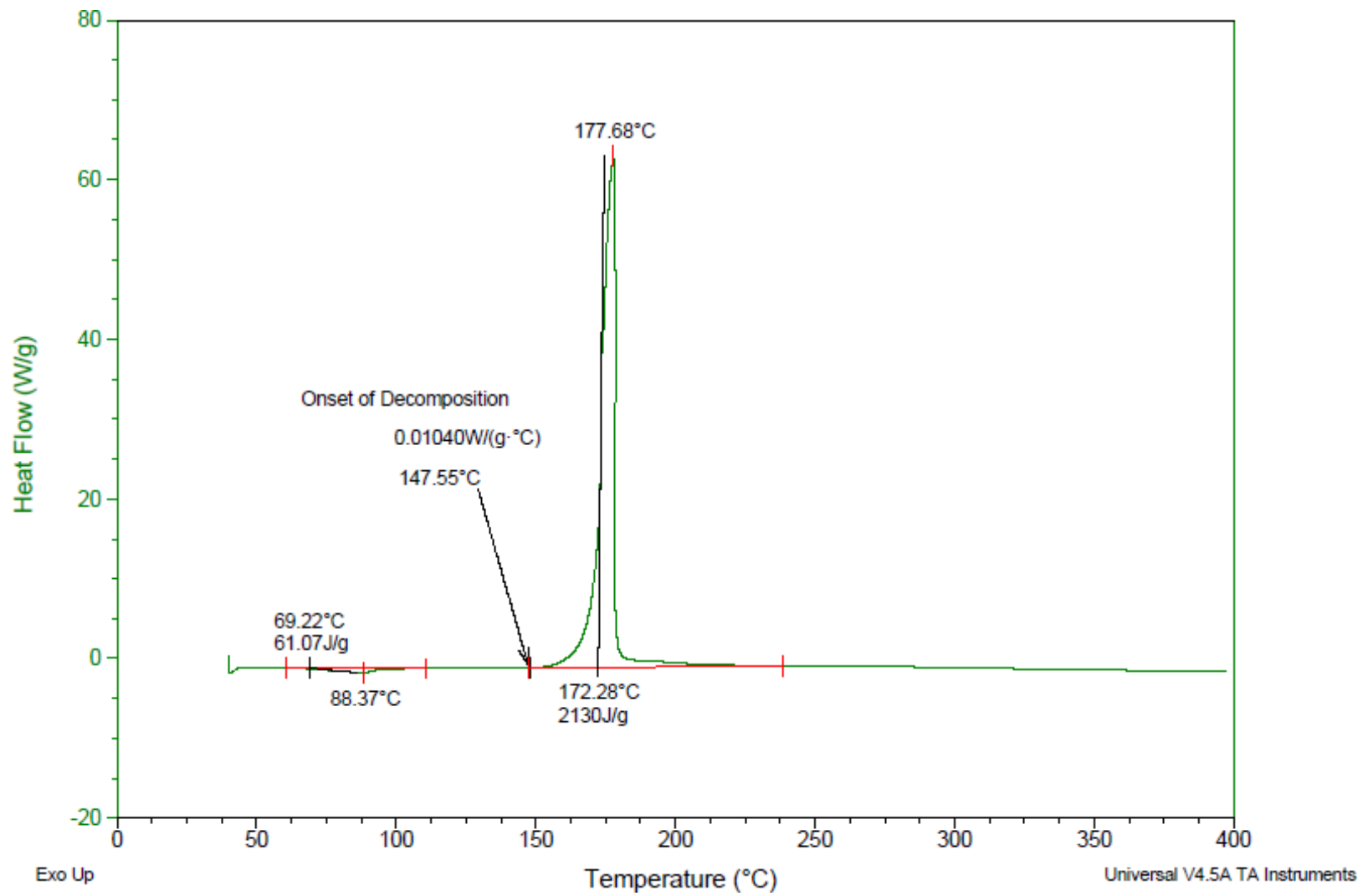

Figure S16: DSC trace of compound (4) 


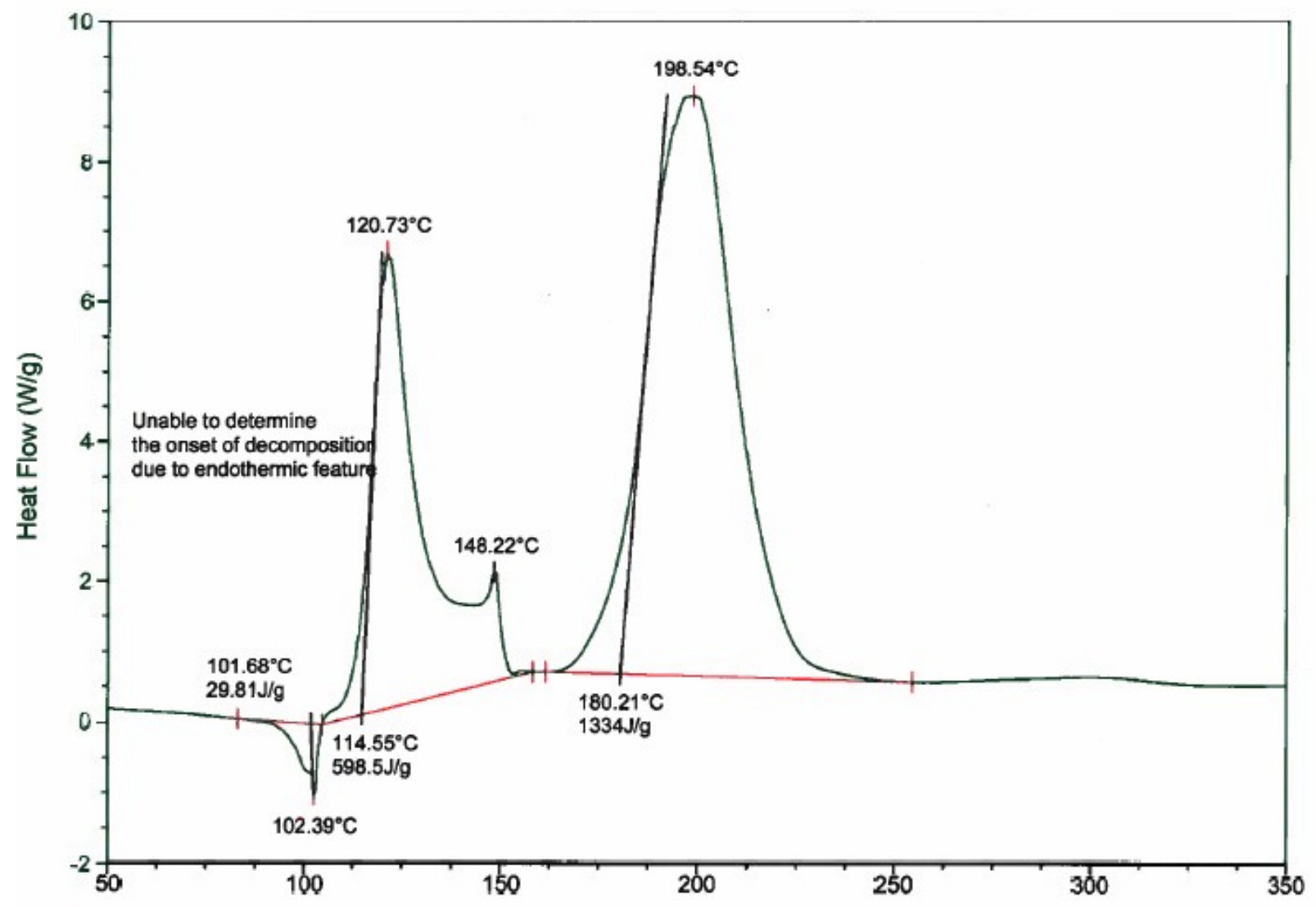

Figure S17: DSC trace of compound (8) 


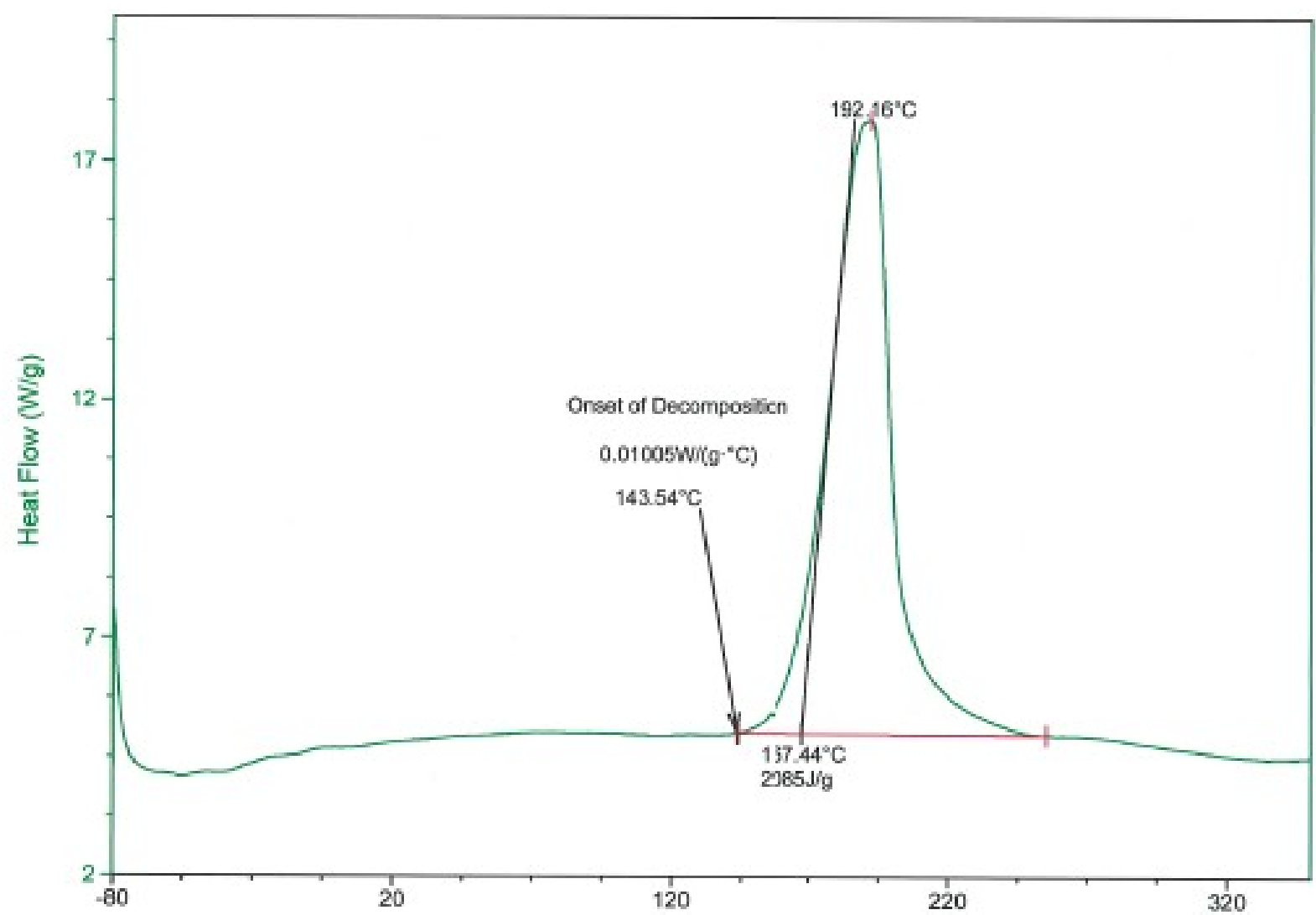

Figure S18: DSC trace of compound (9) 
Thermogravametric analysis mass spectroscopy data:

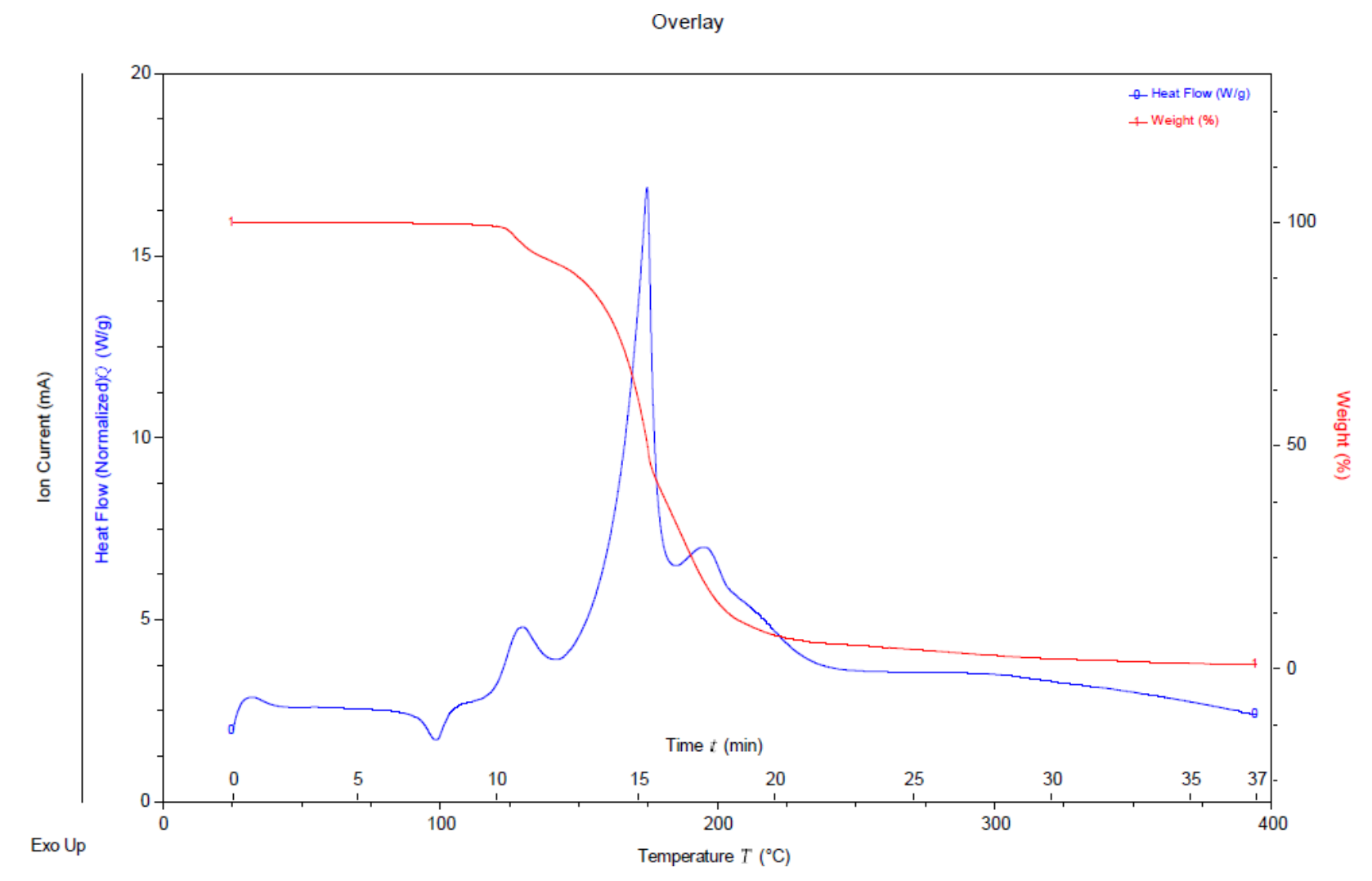

Figure S19: TGA MS of compound (8) 


\section{Crystal Data:}

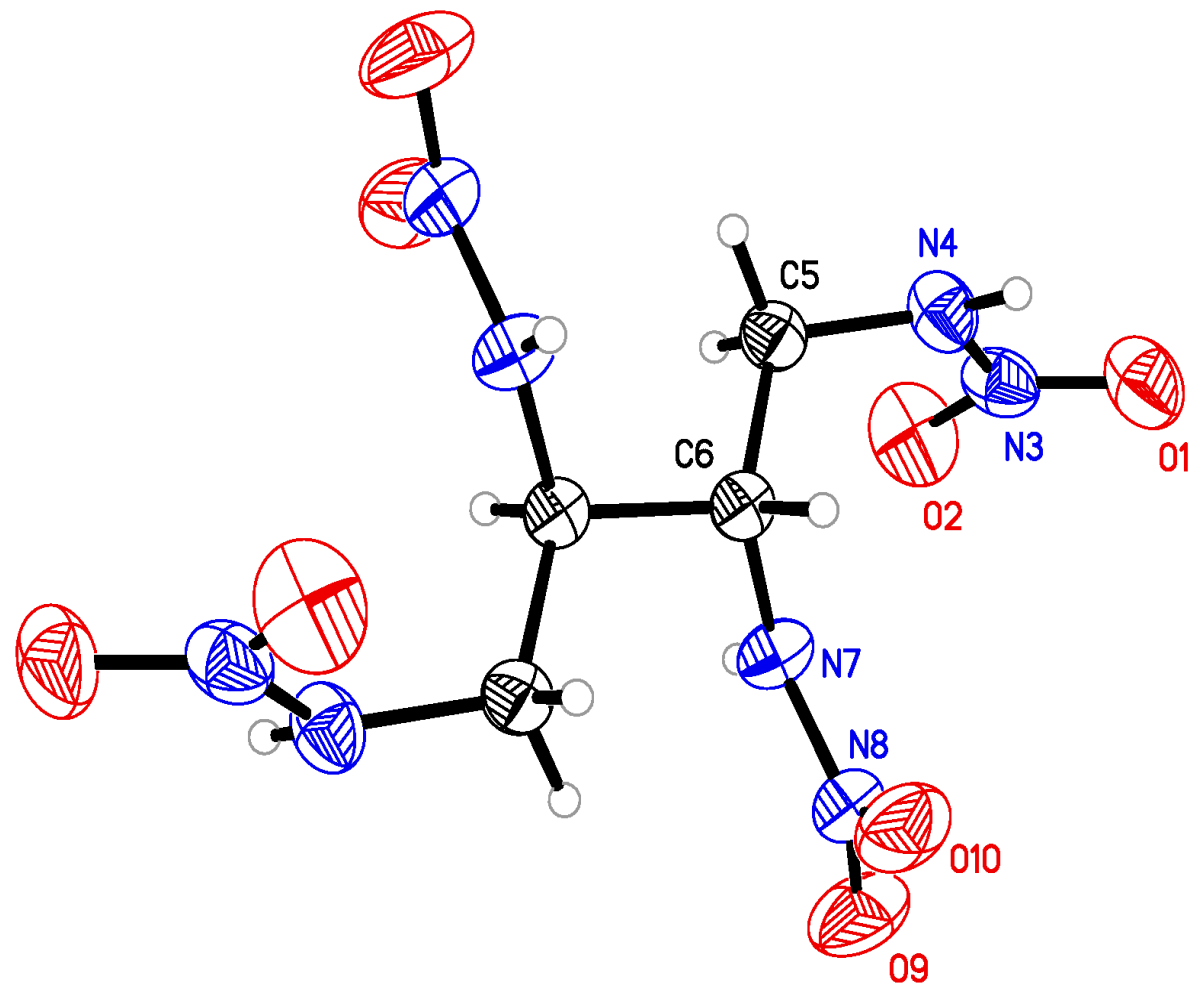

Figure S20: Thermal ellipsoid diagram for Compound (4) with thermal ellipsoids shown at 50\% probability level. 


\section{Crystal Preparation of Compound (4):}

Compound (4) (100mg) was dissolved in the minimal amount of methanol. The solution was transferred to a large beaker. The solution was then allowed to slowly evaporate over multiple days. After 4 days, cubic crystals of compound (4) were obtained.

\section{Single-crystal X-ray Diffraction Analysis of Compound (4).}

$\mathrm{C} 4 \mathrm{H} 10 \mathrm{~N} 8 \mathrm{O} 8, \mathrm{FW}=298.20$, monoclinic, $\mathrm{P} 21 / \mathrm{n}, \mathrm{a}=7.7420$ (19) $\AA, \mathrm{b}=6.4525(13) \AA, \mathrm{c}=11.1204(18) \AA, \alpha=90^{\circ}, \beta=$ $92.759(10)^{\circ}, \gamma=90^{\circ}, V=554.9(2) \AA^{3}, Z=2, \rho_{\text {calc }}(293 \mathrm{~K})=1.785 \mathrm{Mg} / \mathrm{m}^{3}, \mu=1.507 \mathrm{~mm}^{-1}, F(000)=308, \mathrm{R} 1=0.0389$ for 1035 observed $(I>2 \sigma \mathrm{l})$ reflections and 0.0397 for all 1071 reflections, Goodness-of-fit $=1.087,97$ parameters. A clear colorless plate crystal of dimensions $0.270 \times 0.208 \times 0.042$ mmwas mounted on a MiteGen MicroMesh using a small amount of Cargille Immersion Oil. Data were collected on a Bruker three-circle platform diffractometer equipped with a PHOTON 100 CMOS detector. The crystals were irradiated using a $1 \mu$ s microfocus CuKa source $(\lambda=1.54178)$ with Helios optics. Data was collected at room temperature $\left(20^{\circ} \mathrm{C}\right)$. Data collection was performed and the unit cell was initially refined using APEX3 [v2015.5-2]. ${ }^{1}$ Data Reduction was performed using SAINT [v8.34A] ${ }^{2}$ and XPREP [v2014/2] ${ }^{3}$. Corrections were applied for Lorentz, polarization, and absorption effects using SADABS [v2014/2]. ${ }^{4}$ The structure was solved and refined with the aid of the program SHELXL-2014/7. ${ }^{5}$ The full-matrix leastsquares refinement on $\mathrm{F}^{2}$ included atomic coordinates and anisotropic thermal parameters for all non- $\mathrm{H}$ atoms. Hydrogen atoms were located from the difference electron-density maps and added using a riding model.

1. Bruker (2015). APEX3 v2015.5-2. Bruker AXS Inc., Madison, Wisconsin, USA. 2. Bruker (2013). SAINT v8.34A. Bruker AXS Inc., Madison, Wisconsin, USA.

3. Bruker (2014). XPREP v2014/2. Bruker AXS Inc., Madison, Wisconsin, USA.

4. Bruker (2014). SADABS v2014/5, Bruker AXS Inc., Madison, Wisconsin, USA.

5. Sheldrick, G. M. (2014). SHELXL-2014/7. University of Göttingen, Germany. 
Table S9. Crystal data and structure refinement for Compound (4).

Identification code

Empirical formula

Formula weight

Temperature

Wavelength

Crystal system

Space group

Unit cell dimensions

Volume

Z

Density $\left(20^{\circ} \mathrm{C}\right)$

Absorption coefficient

$\mathrm{F}(000)$

Crystal size

Theta range for data collection

Index ranges

Reflections collected

Independent reflections

Completeness to theta $=67.679^{\circ}$

Absorption correction

Max. and min. transmission

Refinement method

Data / restraints / parameters

Goodness-of-fit on $\mathrm{F}^{2}$

Final $R$ indices [ $>2$ sigma(I)]

$R$ indices (all data)

Largest diff. peak and hole
Compound (4)

$\mathrm{C} 4 \mathrm{H} 10 \mathrm{~N} 8 \mathrm{O} 8$

298.20

$566(2) \mathrm{K}$

$1.54178 \AA$

Monoclinic

$\mathrm{P} 21 / \mathrm{n}$

$a=7.7420(19) \AA$

$\alpha=90^{\circ}$.

$b=6.4525(13) \AA$

$\beta=92.759(10)^{\circ}$.

$c=11.1204(18) \AA$

$\mathrm{Y}=90^{\circ}$.

$554.9(2) \AA^{3}$

2

$1.785 \mathrm{Mg} / \mathrm{m}^{3}$

$1.507 \mathrm{~mm}^{-1}$

308

$0.270 \times 0.208 \times 0.042 \mathrm{~mm}^{3}$

6.817 to $74.538^{\circ}$.

$-9<=\mathrm{h}<=9,-7<=\mathrm{k}<=8,-13<=\mathrm{l}<=11$

3997

$1071[$ Rint $=0.0258]$

$97.3 \%$

Semi-empirical from equivalents

0.7538 and 0.5962

Full-matrix least-squares on $\mathrm{F}^{2}$

1071 / 0 / 97

1.087

$\mathrm{R} 1=0.0389, w R 2=0.1017$

$\mathrm{R}_{1}=0.0397, w R_{2}=0.1024$

0.252 and -0.191 e. $\AA^{-3}$ 
Table S10. Atomic coordinates $\left(x 10^{4}\right)$ and equivalent isotropic displacement parameters $\left(\AA^{2} \mathrm{x}\right.$ $10^{3}$ ) for Compound (4). $U(\mathrm{eq})$ is defined as one third of the trace of the orthogonalized $\mathrm{Uij}^{\mathrm{ij}}$ tensor.

\begin{tabular}{lrrrr}
\hline & $\mathrm{x}$ & $\mathrm{y}$ & $\mathrm{z}$ & $\mathrm{U}(\mathrm{eq})$ \\
\hline $\mathrm{O}(1)$ & $510(2)$ & $5178(2)$ & $6408(1)$ & $55(1)$ \\
$\mathrm{O}(2)$ & $1412(2)$ & $7966(2)$ & $7331(1)$ & $58(1)$ \\
$\mathrm{N}(3)$ & $1135(2)$ & $6945(2)$ & $6420(1)$ & $37(1)$ \\
$\mathrm{N}(4)$ & $1472(2)$ & $7744(2)$ & $5348(1)$ & $36(1)$ \\
$\mathrm{C}(5)$ & $2538(2)$ & $9574(2)$ & $5243(1)$ & $31(1)$ \\
$\mathrm{C}(6)$ & $4468(2)$ & $9041(2)$ & $5179(1)$ & $26(1)$ \\
$\mathrm{N}(7)$ & $5062(2)$ & $8269(2)$ & $6353(1)$ & $33(1)$ \\
$\mathrm{N}(8)$ & $6190(2)$ & $6715(2)$ & $6494(1)$ & $38(1)$ \\
$\mathrm{O}(9)$ & $6654(2)$ & $6277(2)$ & $7537(1)$ & $63(1)$ \\
$\mathrm{O}(10)$ & $6686(2)$ & $5845(2)$ & $5595(1)$ & $54(1)$ \\
\hline
\end{tabular}

Table S7. Bond lengths $[\AA]$ and angles $\left[{ }^{\circ}\right]$ for Compound (4).

\begin{tabular}{lc}
\hline $\mathrm{O}(1)-\mathrm{N}(3)$ & $1.2384(19)$ \\
$\mathrm{O}(2)-\mathrm{N}(3)$ & $1.2190(18)$ \\
$\mathrm{N}(3)-\mathrm{N}(4)$ & $1.3356(17)$ \\
$\mathrm{N}(4)-\mathrm{C}(5)$ & $1.4485(19)$ \\
$\mathrm{N}(4)-\mathrm{H}(4)$ & $0.82(2)$ \\
$\mathrm{C}(5)-\mathrm{C}(6)$ & $1.5388(18)$ \\
$\mathrm{C}(5)-\mathrm{H}(5 \mathrm{~A})$ & 0.9700 \\
$\mathrm{C}(5)-\mathrm{H}(5 \mathrm{~B})$ & 0.9700 \\
$\mathrm{C}(6)-\mathrm{N}(7)$ & $1.4512(16)$ \\
$\mathrm{C}(6)-\mathrm{C}(6) \# 1$ & $1.549(3)$ \\
$\mathrm{C}(6)-\mathrm{H}(6)$ & 0.9800 \\
$\mathrm{~N}(7)-\mathrm{N}(8)$ & $1.3341(17)$ \\
$\mathrm{N}(7)-\mathrm{H}(7)$ & $0.91(2)$ \\
$\mathrm{N}(8)-\mathrm{O}(10)$ & $1.2243(18)$ \\
$\mathrm{N}(8)-\mathrm{O}(9)$ & $1.2304(17)$ \\
& \\
$\mathrm{O}(2)-\mathrm{N}(3)-\mathrm{O}(1)$ & $124.03(13)$ \\
$\mathrm{O}(2)-\mathrm{N}(3)-\mathrm{N}(4)$ & $119.95(14)$ \\
$\mathrm{O}(1)-\mathrm{N}(3)-\mathrm{N}(4)$ & $116.00(13)$ \\
$\mathrm{N}(3)-\mathrm{N}(4)-\mathrm{C}(5)$ & $121.54(12)$ \\
$\mathrm{N}(3)-\mathrm{N}(4)-\mathrm{H}(4)$ & $110.6(14)$ \\
$\mathrm{C}(5)-\mathrm{N}(4)-\mathrm{H}(4)$ & $122.4(14)$ \\
$\mathrm{N}(4)-\mathrm{C}(5)-\mathrm{C}(6)$ & $112.33(12)$ \\
$\mathrm{N}(4)-\mathrm{C}(5)-\mathrm{H}(5 \mathrm{~A})$ & 109.1 \\
$\mathrm{C}(6)-\mathrm{C}(5)-\mathrm{H}(5 \mathrm{~A})$ & 109.1 \\
&
\end{tabular}




$\begin{array}{ll}\mathrm{N}(4)-\mathrm{C}(5)-\mathrm{H}(5 \mathrm{~B}) & 109.1 \\ \mathrm{C}(6)-\mathrm{C}(5)-\mathrm{H}(5 \mathrm{~B}) & 109.1 \\ \mathrm{H}(5 \mathrm{~A})-\mathrm{C}(5)-\mathrm{H}(5 \mathrm{~B}) & 107.9 \\ \mathrm{~N}(7)-\mathrm{C}(6)-\mathrm{C}(5) & 107.55(11) \\ \mathrm{N}(7)-\mathrm{C}(6)-\mathrm{C}(6) \# 1 & 110.90(13) \\ \mathrm{C}(5)-\mathrm{C}(6)-\mathrm{C}(6) \# 1 & 111.29(13) \\ \mathrm{N}(7)-\mathrm{C}(6)-\mathrm{H}(6) & 109.0 \\ \mathrm{C}(5)-\mathrm{C}(6)-\mathrm{H}(6) & 109.0 \\ \mathrm{C}(6) \# 1-\mathrm{C}(6)-\mathrm{H}(6) & 109.0 \\ \mathrm{~N}(8)-\mathrm{N}(7)-\mathrm{C}(6) & 122.74(12) \\ \mathrm{N}(8)-\mathrm{N}(7)-\mathrm{H}(7) & 112.3(13) \\ \mathrm{C}(6)-\mathrm{N}(7)-\mathrm{H}(7) & 124.4(13) \\ \mathrm{O}(10)-\mathrm{N}(8)-\mathrm{O}(9) & 125.15(14) \\ \mathrm{O}(10)-\mathrm{N}(8)-\mathrm{N}(7) & 118.55(13) \\ \mathrm{O}(9)-\mathrm{N}(8)-\mathrm{N}(7) & 116.30(14)\end{array}$

Symmetry transformations used to generate equivalent atoms:

$\# 1-x+1,-y+2,-z+1$ 
Table S11. Anisotropic displacement parameters $\left(\AA^{2} \times 10^{3}\right)$ for Compound (4). The anisotropic displacement factor exponent takes the form: $-2 \pi^{2}\left[h^{2} a^{* 2} U^{11}+\ldots+2 h k a^{*} b^{*} U^{12}\right]$

\begin{tabular}{lllllll}
\hline & $\mathrm{U}^{11}$ & $\mathrm{U}^{22}$ & $\mathrm{U} 33$ & $\mathrm{U} 23$ & $\mathrm{U}^{13}$ & $\mathrm{U}^{12}$ \\
\hline $\mathrm{O}(1)$ & $57(1)$ & $61(1)$ & $45(1)$ & $17(1)$ & $-5(1)$ & $-25(1)$ \\
$\mathrm{O}(2)$ & $68(1)$ & $74(1)$ & $34(1)$ & $-6(1)$ & $13(1)$ & $-11(1)$ \\
$\mathrm{N}(3)$ & $28(1)$ & $51(1)$ & $33(1)$ & $7(1)$ & $2(1)$ & $-4(1)$ \\
$\mathrm{N}(4)$ & $36(1)$ & $41(1)$ & $30(1)$ & $1(1)$ & $3(1)$ & $-9(1)$ \\
$\mathrm{C}(5)$ & $28(1)$ & $32(1)$ & $33(1)$ & $0(1)$ & $2(1)$ & $0(1)$ \\
$\mathrm{C}(6)$ & $28(1)$ & $26(1)$ & $24(1)$ & $-1(1)$ & $2(1)$ & $1(1)$ \\
$\mathrm{N}(7)$ & $36(1)$ & $34(1)$ & $27(1)$ & $4(1)$ & $3(1)$ & $9(1)$ \\
$\mathrm{N}(8)$ & $37(1)$ & $31(1)$ & $45(1)$ & $5(1)$ & $-2(1)$ & $4(1)$ \\
$\mathrm{O}(9)$ & $75(1)$ & $60(1)$ & $52(1)$ & $20(1)$ & $-14(1)$ & $18(1)$ \\
$\mathrm{O}(10)$ & $55(1)$ & $44(1)$ & $62(1)$ & $-12(1)$ & $-2(1)$ & $20(1)$ \\
\hline
\end{tabular}

Table S12. Hydrogen coordinates $\left(x 10^{4}\right)$ and isotropic displacement parameters $\left(\AA^{2} \times 10^{3}\right)$ for Compound (4).

\begin{tabular}{lllll}
\hline & \multicolumn{1}{c}{$\mathrm{x}$} & \multicolumn{1}{c}{$\mathrm{y}$} & $\mathrm{Z}$ & $\mathrm{U}(\mathrm{eq})$ \\
\hline $\mathrm{H}(4)$ & $1400(30)$ & $6850(30)$ & $4823(19)$ & 54 \\
$\mathrm{H}(5 \mathrm{~A})$ & 2381 & 10468 & 5931 & 37 \\
$\mathrm{H}(5 \mathrm{~B})$ & 2162 & 10331 & 4524 & 37 \\
$\mathrm{H}(6)$ & 4601 & 7947 & 4580 & 31 \\
$\mathrm{H}(7)$ & $4840(30)$ & $8890(30)$ & $7063(18)$ & 49 \\
\hline
\end{tabular}


Table S13. Torsion angles $\left[{ }^{\circ}\right]$ for Compound (4).

\begin{tabular}{lc}
\hline $\mathrm{O}(2)-\mathrm{N}(3)-\mathrm{N}(4)-\mathrm{C}(5)$ & $15.2(2)$ \\
$\mathrm{O}(1)-\mathrm{N}(3)-\mathrm{N}(4)-\mathrm{C}(5)$ & $-166.27(13)$ \\
$\mathrm{N}(3)-\mathrm{N}(4)-\mathrm{C}(5)-\mathrm{C}(6)$ & $89.14(16)$ \\
$\mathrm{N}(4)-\mathrm{C}(5)-\mathrm{C}(6)-\mathrm{N}(7)$ & $-69.41(14)$ \\
$\mathrm{N}(4)-\mathrm{C}(5)-\mathrm{C}(6)-\mathrm{C}(6) \# 1$ & $168.94(13)$ \\
$\mathrm{C}(5)-\mathrm{C}(6)-\mathrm{N}(7)-\mathrm{N}(8)$ & $141.96(13)$ \\
$\mathrm{C}(6) \# 1-\mathrm{C}(6)-\mathrm{N}(7)-\mathrm{N}(8)$ & $-96.15(17)$ \\
$\mathrm{C}(6)-\mathrm{N}(7)-\mathrm{N}(8)-\mathrm{O}(10)$ & $-3.4(2)$ \\
$\mathrm{C}(6)-\mathrm{N}(7)-\mathrm{N}(8)-\mathrm{O}(9)$ & $176.48(14)$
\end{tabular}

Symmetry transformations used to generate equivalent atoms:

$\# 1-x+1,-y+2,-z+1$

Table S14. Hydrogen bonds for Compound (4) [ $\AA$ and $\left.{ }^{\circ}\right]$.

\begin{tabular}{lllll}
\hline \multicolumn{1}{c}{$\mathrm{D}-\mathrm{H} \ldots \mathrm{A}$} & $\mathrm{d}(\mathrm{D}-\mathrm{H})$ & $\mathrm{d}(\mathrm{H} \ldots \mathrm{A})$ & $\mathrm{d}(\mathrm{D} \ldots \mathrm{A})$ & $<(\mathrm{DHA})$ \\
\hline $\mathrm{N}(4)-\mathrm{H}(4) \ldots \mathrm{O}(1) \# 2$ & $0.82(2)$ & $2.36(2)$ & $3.0712(18)$ & $145.0(19)$ \\
$\mathrm{N}(4)-\mathrm{H}(4) \ldots \mathrm{O}(10) \# 3$ & $0.82(2)$ & $2.34(2)$ & $2.9398(19)$ & $130.0(19)$ \\
$\mathrm{N}(7)-\mathrm{H}(7) \ldots \mathrm{O}(1) \# 4$ & $0.91(2)$ & $1.92(2)$ & $2.8319(17)$ & $177.3(19)$
\end{tabular}

Symmetry transformations used to generate equivalent atoms:

$\# 1-x+1,-y+2,-z+1 \quad \# 2-x,-y+1,-z+1 \quad \# 3-x+1,-y+1,-z+1$

$\# 4-x+1 / 2, y+1 / 2,-z+3 / 2 \quad \# 5 x-1 / 2,-y+3 / 2, z-1 / 2$ 